\title{
Supporting Information for Computational Material Screening using Artificial Neural Networks for Adsorption Gas Separation
}

\author{
Akhil Arora, Shachit S. Iyer, M. M. Faruque Hasan* \\ Artie McFerrin Department of Chemical Engineering, Texas A\&M University \\ College Station, TX 77843-3122, USA.
}

\section{Contents}

S1 First principles-based Adsorption Model $\quad$ S2

S2 Parameters used for Breakthrough Validation $\quad$ S3

S3 Effect of Material Properties on Breakthrough Dynamics S4

S4 ANN Cross Validation $\quad$ S6

S5 Fitted Adsorption Isotherm Parameters $\quad$ S7

$\mathrm{S} 5.1 \mathrm{CO}_{2}$ adsorption isotherm parameters on dual-site model . . . . . . . . . . . . S7

$\mathrm{S} 5.2 \mathrm{~N}_{2}$ adsorption isotherm parameters on single-site model . . . . . . . . . . . S11

S5.3 $\mathrm{CH}_{4}$ adsorption isotherm parameters on single-site model . . . . . . . . . . S16

S6 ANN Model Performance Comparison $\quad$ S20

$\begin{array}{ll}\text { S7 Material Rankings } & \text { S21 }\end{array}$

S7.1 Performance metrics for $\mathrm{CO}_{2} / \mathrm{N}_{2}$ separation . . . . . . . . . . . . . . S21

S7.2 Performance metrics for $\mathrm{CO}_{2} / \mathrm{CH}_{4}$ separation . . . . . . . . . . . . . S25 


\section{S1 First principles-based Adsorption Model}

For a species $i$ in the gas phase, the component mass balance equation is as follows:

$$
\varepsilon_{t} \frac{\partial C_{i}}{\partial t}=\varepsilon_{b} \frac{\partial}{\partial z}\left(D_{L} C \frac{\partial y_{i}}{\partial z}\right)-\varepsilon_{b} \frac{\partial\left(v C_{i}\right)}{\partial z}-\rho_{b, a d s} \frac{\partial \bar{q}_{i}}{\partial t}
$$

where $C_{i}$ and $C$ are individual species and overall gas phase concentration, respectively, $y_{i}$ is the gas phase mole fraction, $v$ is the interstitial velocity, $\varepsilon_{b}$ and $\varepsilon_{t}$ are the bed and total void fraction, $\rho_{b, a d s}$ is the adsorbent bulk density, $\bar{q}_{i}$ is the solid phase concentration of species $i, D_{L}$ is the axial dispersion coefficient, and $z$ and $t$ are respectively the space and time coordinates.

The ideal gas law is then used for converting gas phase concentrations, $C_{i}$ and $C$, to mole fraction $y_{i}$ as follows:

$$
\frac{\partial y_{i}}{\partial t}+\frac{y_{i}}{P} \frac{\partial P}{\partial t}-\frac{y_{i}}{T} \frac{\partial T}{\partial t}=D_{L} \frac{\varepsilon_{b}}{\varepsilon_{t}} \frac{T}{P} \frac{\partial}{\partial z}\left(\frac{P}{T} \frac{\partial y_{i}}{\partial z}\right)-\frac{\varepsilon_{b}}{\varepsilon_{t}} \frac{T}{P} \frac{\partial}{\partial z}\left(\frac{y_{i} v P}{T}\right)-\frac{\rho_{b, a d s} R T}{\varepsilon_{t} P} \frac{\partial \bar{q}_{i}}{\partial t}
$$

where $P$ and $T$ are the gas phase pressure and temperature, respectively, and $R$ is the universal gas constant.

The overall mass balance equation is obtained by summing over Eq. 1b for all species $i \in I$ as follows:

$$
\frac{\partial P}{\partial t}=\frac{P}{T} \frac{\partial T}{\partial t}-\frac{\varepsilon_{b} T}{\varepsilon_{t}} \frac{\partial}{\partial z}\left(\frac{P v}{T}\right)-\frac{\rho_{b, a d s} R T}{\varepsilon_{t}} \sum_{i \in I} \frac{\partial \overline{q_{i}}}{\partial t}
$$

To consider the heat effects inside the adsorption column, the overall heat balance expression for a bed volume element is as follows:

$$
\begin{gathered}
\left(\rho_{b, a d s} C_{p, a d s}+C_{p, a} \rho_{b, a d s} \sum_{i \in I} \bar{q}_{i}\right) \\
\frac{\partial T}{\partial t}=K_{z} \frac{\partial^{2} T}{\partial z^{2}}-\frac{C_{p g} \varepsilon_{b}}{R} \frac{\partial}{\partial z}(v P)-C_{p, a} \rho_{b, a d s} T \sum_{i \in I} \frac{\partial \bar{q}_{i}}{\partial t}-\frac{C_{p g} \varepsilon_{t}}{R} \frac{\partial P}{\partial t} \\
+\rho_{b, a d s} \sum_{i \in I}\left(-\Delta H_{i}\right) \frac{\partial \bar{q}_{i}}{\partial t}-\frac{2 h_{i n}}{r_{i n}}\left(T-T_{w}\right)
\end{gathered}
$$

where $T$ is the gas phase temperature, $T_{w}$ is the wall temperature, $C_{p, a}$ and $C_{p, a d s}$ are respectively the adsorbate and adsorbent specific heat capacities, $C_{p g}$ is the heat capacity of gas mixture, $K_{z}$ is the axial heat conductivity, $r_{i n}$ is the internal bed radius and $h_{i n}$ is the internal heat transfer coefficient.

To consider pressure drop inside adsorber column, the Darcy's law equation is used for describing the steady-state momentum balance as follows:

$$
-\frac{\partial P}{\partial z}=\frac{150}{4 r_{p}^{2}}\left(\frac{1-\varepsilon_{b}}{\varepsilon_{b}}\right)^{2} \mu v
$$

where $r_{p}$ is the particle radius and $\mu$ is the gas mixture viscosity. 
For describing the mass transfer of adsorbate from gas to solid phase, the following linear driving force (LDF) model is used:

$$
\frac{\partial \overline{q_{i}}}{\partial t}=k_{i}\left(q_{i}^{*}-\overline{q_{i}}\right)
$$

where $q_{i}^{*}$ and $\bar{q}_{i}$ are respectively the equilibrium and actual adsorbent loading capacities, and $k_{i}$ is the lumped mass transfer coefficient for species $i$.

\section{S2 Parameters used for Breakthrough Validation}

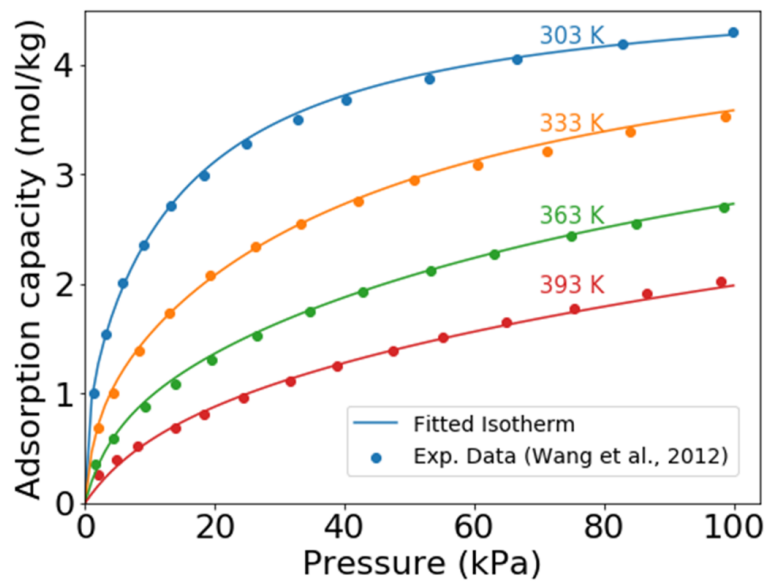

(a)

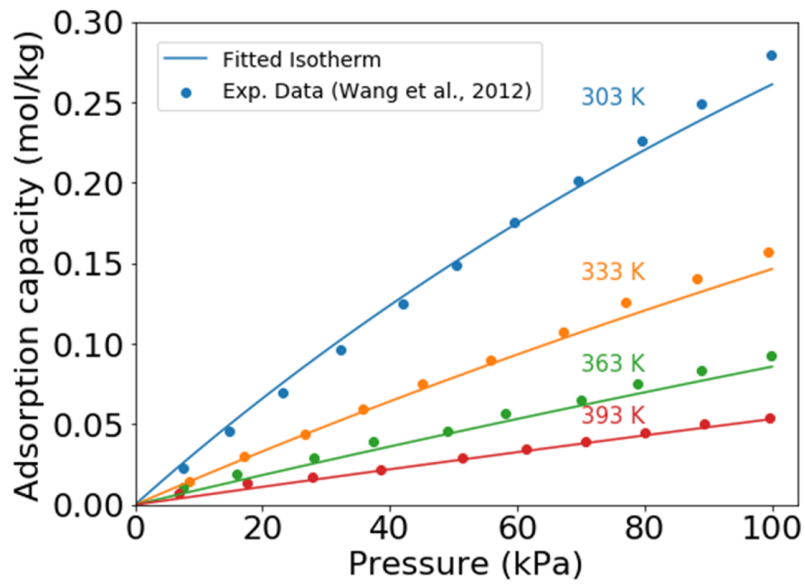

(b)

Figure S1: Fitted dual site adsorption isotherms for (a) $\mathrm{CO}_{2}$ and (b) $\mathrm{N}_{2}$ adsorption on zeolite 13X-APG.

Table S1: Fitted dual site isotherm parameters for $\mathrm{CO}_{2}$ and $\mathrm{N}_{2}$ adsorption on zeolite 13XAPG.

\begin{tabular}{ccccccc}
\hline Gas species $(i)$ & $\begin{array}{c}m_{i, 1} \\
(\mathrm{~mol} / \mathrm{kg})\end{array}$ & $\begin{array}{c}m_{i, 2} \\
(\mathrm{~mol} / \mathrm{kg})\end{array}$ & $\begin{array}{c}b_{o, i, 1} \\
\left(\mathrm{~Pa}^{-1}\right)\end{array}$ & $\begin{array}{c}b_{o, i, 2} \\
\left(\mathrm{~Pa}^{-1}\right)\end{array}$ & $\begin{array}{c}\Delta U_{i, 1} \\
(\mathrm{~J} / \mathrm{mol})\end{array}$ & $\begin{array}{c}\Delta U_{i, 1} \\
(\mathrm{~J} / \mathrm{mol})\end{array}$ \\
\hline $\mathrm{CO}_{2}$ & 3.7854 & 1.0005 & $3.92 \times 10^{-10}$ & $5.17 \times 10^{-10}$ & -30266.91 & -38695.35 \\
$\mathrm{~N}_{2}$ & 1 & 0.4260 & $1.16 \times 10^{-9}$ & 0 & -20207.66 & -25944.05 \\
\hline
\end{tabular}


Table S2: Simulation parameters for validating breakthrough concentration profiles.

\begin{tabular}{llr}
\hline Parameter & Unit & Value \\
\hline Column radius [1] & $\mathrm{m}$ & 0.0125 \\
Column length [1] & $\mathrm{m}$ & 0.35 \\
Bed porosity [1] & - & 0.39 \\
Adsorbent bulk density [1] & $\mathrm{kg} \mathrm{ads.}^{-3}$ bed & 666.5 \\
Axial dispersion coefficient [1] & $\mathrm{m}^{2} \mathrm{~s}^{-1}$ & $8.33 \times 10^{-4}$ \\
Overall heat transfer coefficient [1] & $\mathrm{W} \mathrm{m}^{-2} \mathrm{~K}^{-1}$ & 50 \\
Wall thickness [1] & $\mathrm{m}^{-3}$ & 0.0035 \\
Wall density [1] & $\mathrm{kg} \mathrm{m}^{-3}$ & 8238 \\
Wall heat capacity [1] & $\mathrm{J} \mathrm{kg}^{-1} \mathrm{~K}^{-1}$ & 500 \\
Adsorbent heat capacity [1] & $\mathrm{J} \mathrm{kg}^{-1} \mathrm{~K}^{-1}$ & 920 \\
CO ${ }_{2}$ feed composition [1] & $\%$ & 15 \\
Interstitial velocity [1] & $\mathrm{m} \mathrm{s}^{-1}$ & 0.161 \\
Feed pressure [1] & $\mathrm{bar}^{-1}$ & 1.33 \\
Feed temperature [1] & $\mathrm{K}^{-1}$ & 303 \\
CO ${ }_{2}$ LDF mass transfer coefficient [2] & $\mathrm{s}^{-1}$ & 0.011 \\
$\mathrm{~N}_{2}$ LDF mass transfer coefficient [2] & $\mathrm{s}^{-1}$ & 0.0143 \\
\hline
\end{tabular}

\section{S3 Effect of Material Properties on Breakthrough Dy- namics}

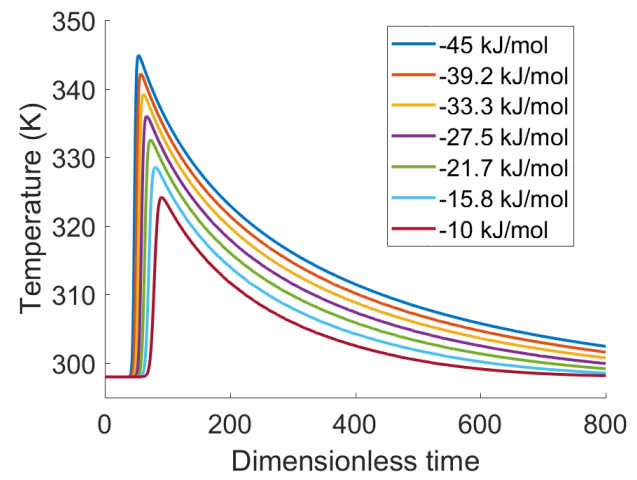

(a)

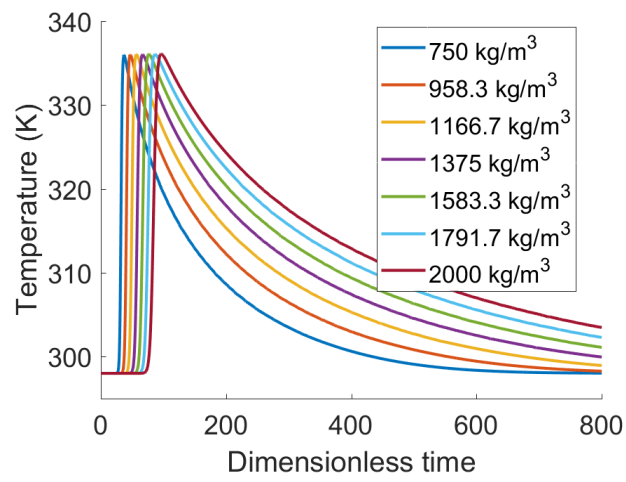

(b)

Figure S2: Effect of (a) heat of adsorption and (b) adsorbent framework density on outlet temperature. 


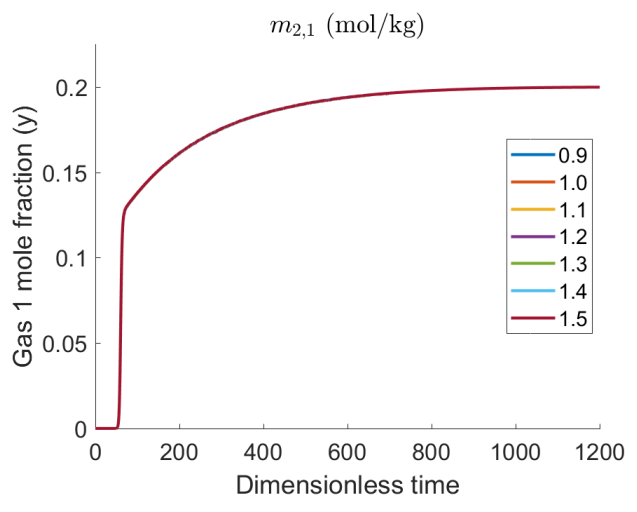

(a)

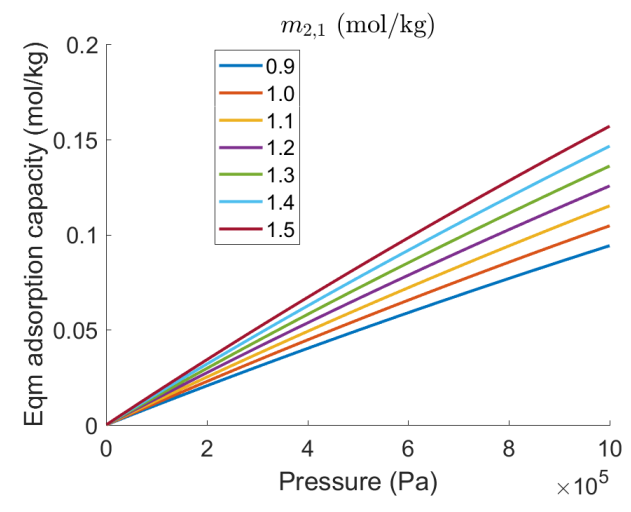

(b)

Figure S3: Effect of saturation adsorption capacity of gas species 2 on (a) breakthrough dynamics and (b) equilibrium adsorption isotherms. Fixed material parameters: $m_{1,1}=m_{1,2}$ $=4 \mathrm{~mol} \mathrm{~kg}{ }^{-1}, b_{1,1}=b_{1,2}=b_{2,1}=10^{-10} \mathrm{~Pa}^{-1}, \Delta U_{1,1}=\Delta U_{1,2}=\Delta H_{1}=-27.5 \mathrm{~kJ} \mathrm{~mol}^{-1}$, $\Delta U_{2,1}=\Delta H_{2}=-17.5 \mathrm{~kJ} \mathrm{~mol}^{-1}, \rho_{b}=1375 \mathrm{~kg}$ ads. $\mathrm{m}^{-3}$ bed.

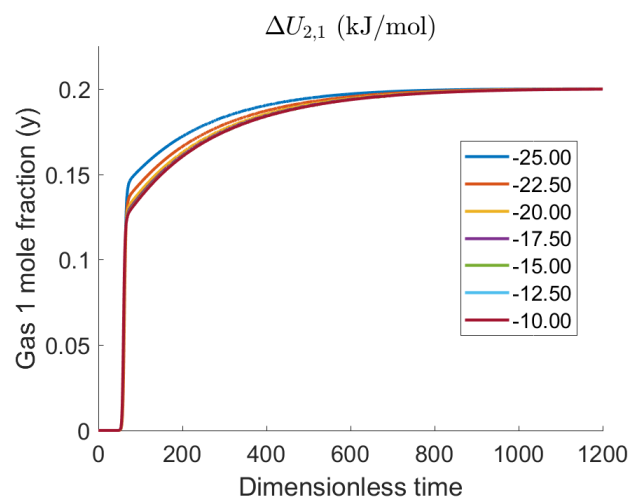

(a)

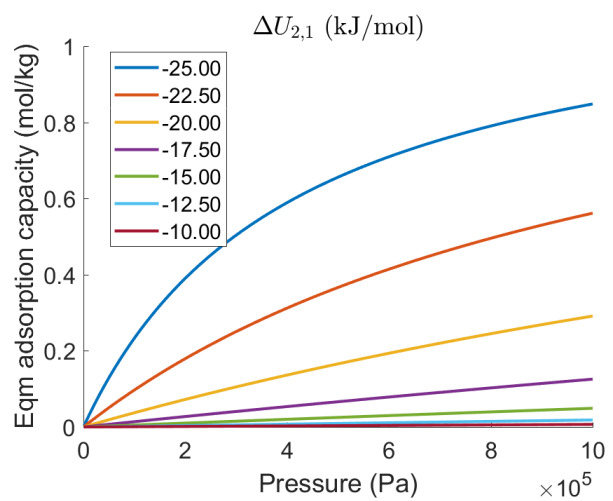

(b)

Figure S4: Effect of adsorption energy of gas 2 on (a) breakthrough dynamics and (b) equilibrium adsorption isotherms. Fixed material parameters: $m_{1,1}=m_{1,2}=4 \mathrm{~mol} \mathrm{~kg}^{-1}$, $b_{1,1}=b_{1,2}=b_{2,1}=10^{-10} \mathrm{~Pa}^{-1}, m_{2,1}=1.2 \mathrm{~mol} \mathrm{~kg}{ }^{-1}, \Delta U_{1,1}=\Delta U_{1,2}=\Delta H_{1}=-27.5 \mathrm{~kJ}$ $\mathrm{mol}^{-1}, \Delta H_{2}=-17.5 \mathrm{~kJ} \mathrm{~mol}^{-1}, \rho_{b}=1375 \mathrm{~kg}$ ads. $\mathrm{m}^{-3}$ bed. 


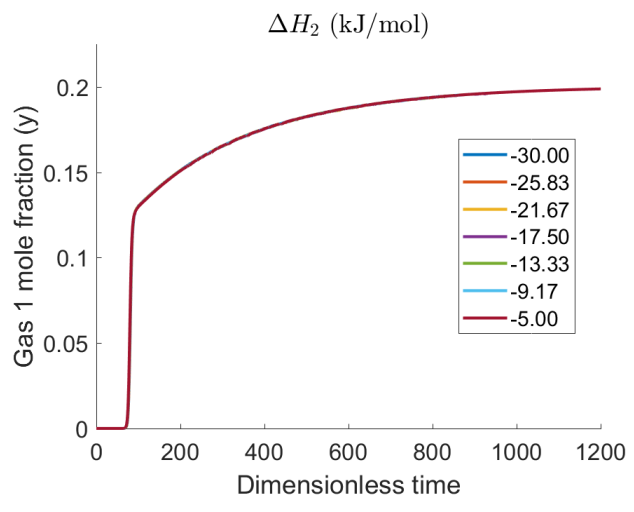

(a)

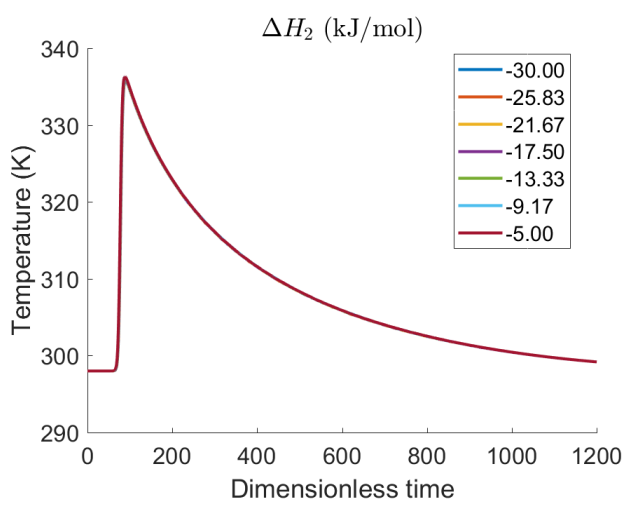

(b)

Figure S5: Effect of heat of adsorption of gas 2 on outlet (a) concentration and (b) temperature profiles. Fixed material parameters: $m_{1,1}=m_{1,2}=4 \mathrm{~mol} \mathrm{~kg}^{-1}, b_{1,1}=b_{1,2}=b_{2,1}=10^{-10}$ $\mathrm{Pa}^{-1}, m_{2,1}=1.2 \mathrm{~mol} \mathrm{~kg}{ }^{-1}, \Delta U_{1,1}=\Delta U_{1,2}=\Delta H_{1}=-27.5 \mathrm{~kJ} \mathrm{~mol}^{-1}, \Delta U_{2,1}=\Delta H_{2}=-17.5$ $\mathrm{kJ} \mathrm{mol}^{-1}, \rho_{b}=1375 \mathrm{~kg}$ ads. $\mathrm{m}^{-3}$ bed.

\section{S4 ANN Cross Validation}
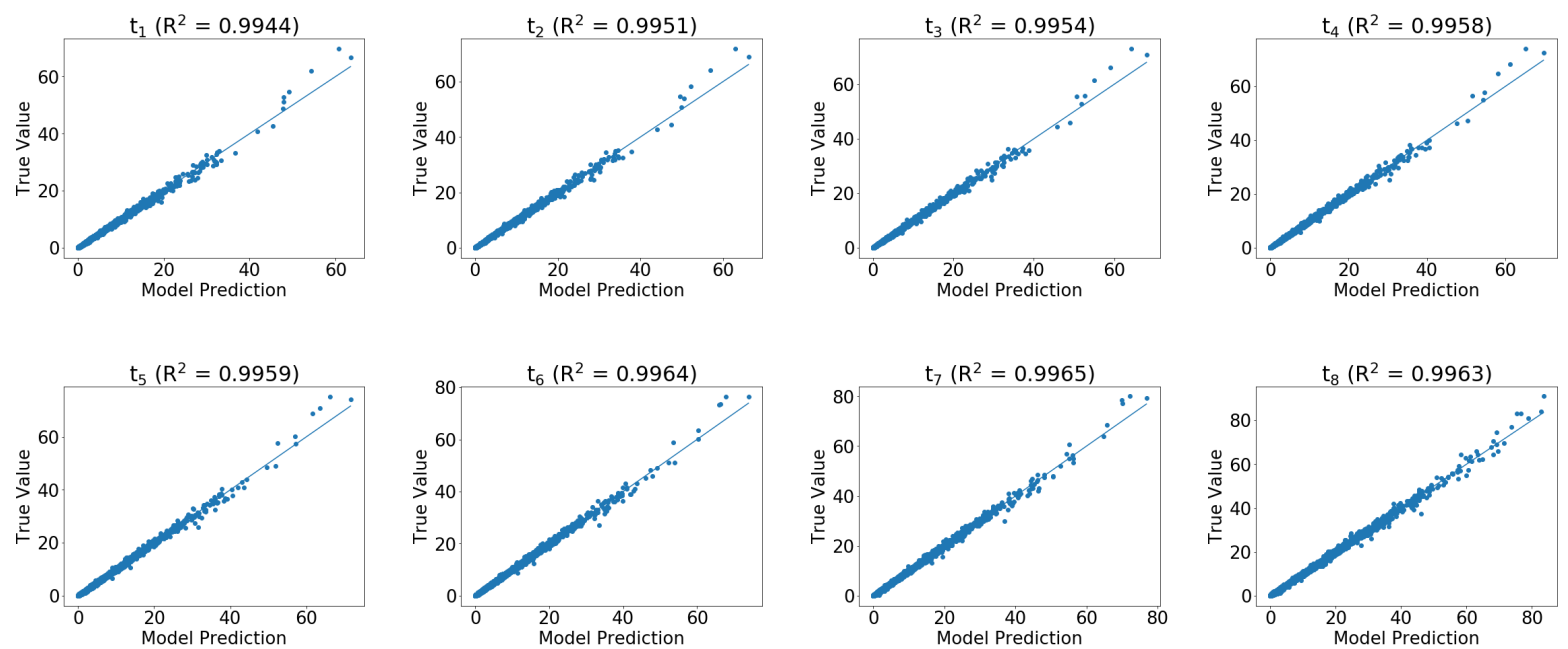

Figure S6: Cross-validating breakthrough time events predictions with 5000 unseen samples for post-combustion carbon capture. 

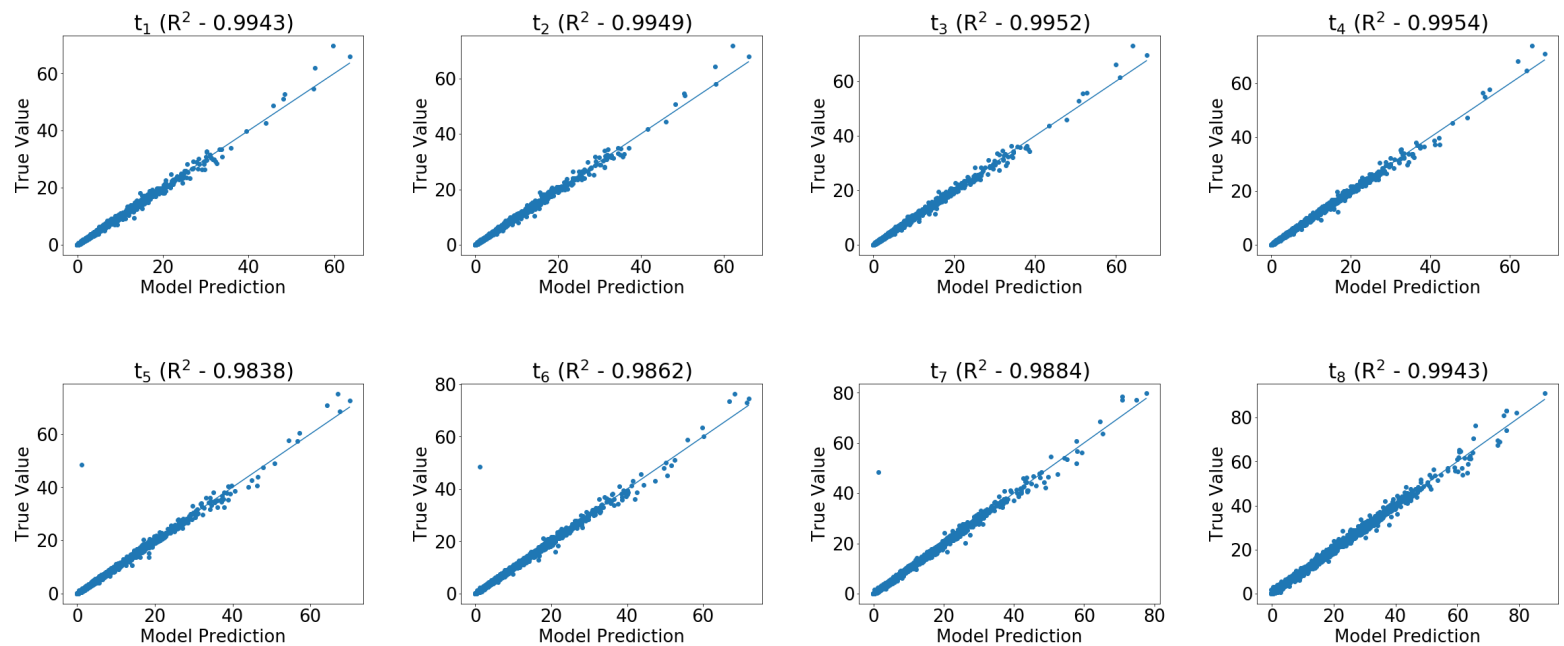

Figure S7: Cross-validating breakthrough time events predictions with 5000 unseen samples for natural gas purification.

\section{S5 Fitted Adsorption Isotherm Parameters}

S5.1 $\mathrm{CO}_{2}$ adsorption isotherm parameters on dual-site model

\begin{tabular}{ccccccc}
\hline Adsorbent & $\begin{array}{c}m_{1,1} \\
\left(\mathrm{~mol} \mathrm{~kg}^{-1}\right)\end{array}$ & $\begin{array}{c}m_{1,2} \\
\left(\mathrm{~mol} \mathrm{~kg}^{-1}\right)\end{array}$ & $\begin{array}{c}b_{1,1} \\
\left(\mathrm{~Pa}^{-1}\right)\end{array}$ & $\begin{array}{c}b_{1,2} \\
\left(\mathrm{~Pa}^{-1}\right)\end{array}$ & $\begin{array}{c}\Delta U_{1,1} \\
\left(\mathrm{~kJ} \mathrm{~mol}^{-1}\right)\end{array}$ & $\begin{array}{c}\Delta U_{1,2} \\
\left(\mathrm{~kJ} \mathrm{~mol}^{-1}\right)\end{array}$ \\
\hline ABW & 1.05 & 5.21 & $1.00 \mathrm{E}-10$ & $1.00 \mathrm{E}-10$ & -17541.79 & -28774.12 \\
ACO & 2.83 & 2.25 & $1.00 \mathrm{E}-10$ & $1.00 \mathrm{E}-10$ & -27652.89 & -20959.07 \\
AEI & 1.00 & 6.79 & $1.00 \mathrm{E}-10$ & $1.00 \mathrm{E}-10$ & -25266.64 & -25266.64 \\
AEL & 1.00 & 1.68 & $1.00 \mathrm{E}-10$ & $1.00 \mathrm{E}-10$ & -15000.00 & -26332.53 \\
AEN & 1.39 & 1.65 & $1.00 \mathrm{E}-10$ & $1.00 \mathrm{E}-10$ & -29025.18 & -29025.18 \\
AET & 4.51 & 3.36 & $1.00 \mathrm{E}-10$ & $1.00 \mathrm{E}-10$ & -15000.00 & -21183.80 \\
AFG & 4.20 & 1.00 & $1.00 \mathrm{E}-10$ & $1.00 \mathrm{E}-10$ & -28804.09 & -18893.41 \\
AFI & 1.00 & 3.81 & $1.00 \mathrm{E}-10$ & $1.00 \mathrm{E}-10$ & -16836.16 & -28358.36 \\
AFN & 3.84 & 1.00 & $1.00 \mathrm{E}-10$ & $1.00 \mathrm{E}-10$ & -28339.74 & -16516.01 \\
AFO & 1.79 & 1.00 & $1.00 \mathrm{E}-10$ & $1.00 \mathrm{E}-10$ & -26477.83 & -15000.00 \\
AFR & 1.98 & 5.48 & $1.00 \mathrm{E}-10$ & $1.00 \mathrm{E}-10$ & -24020.76 & -24020.76 \\
AFS & 5.80 & 2.42 & $1.00 \mathrm{E}-10$ & $1.00 \mathrm{E}-10$ & -24120.49 & -24120.49 \\
AFT & 6.03 & 1.00 & $1.00 \mathrm{E}-10$ & $1.00 \mathrm{E}-10$ & -25088.35 & -29495.06 \\
AFX & 1.00 & 5.98 & $1.00 \mathrm{E}-10$ & $1.00 \mathrm{E}-10$ & -29646.77 & -25154.31 \\
AFY & 1.00 & 6.97 & $1.00 \mathrm{E}-10$ & $1.00 \mathrm{E}-10$ & -26725.92 & -26725.92 \\
AHT & 2.00 & 1.00 & $1.00 \mathrm{E}-10$ & $1.00 \mathrm{E}-10$ & -29808.82 & -29808.82 \\
ANA & 1.00 & 1.00 & $1.00 \mathrm{E}-10$ & $1.00 \mathrm{E}-10$ & -15000.00 & -17042.42 \\
APC & 1.13 & 3.68 & $1.00 \mathrm{E}-10$ & $1.00 \mathrm{E}-10$ & -28778.20 & -28778.20 \\
APD & 2.17 & 1.84 & $1.00 \mathrm{E}-10$ & $1.00 \mathrm{E}-10$ & -30023.94 & -22594.30 \\
AST & 4.89 & 3.24 & $1.00 \mathrm{E}-10$ & $1.00 \mathrm{E}-10$ & -15000.00 & -25089.21 \\
ASV & 1.00 & 2.72 & $1.00 \mathrm{E}-10$ & $1.00 \mathrm{E}-10$ & -15000.00 & -29554.91 \\
& & & & & &
\end{tabular}




\begin{tabular}{|c|c|c|c|c|c|c|}
\hline $\mathrm{ATN}$ & 1.84 & 2.51 & $1.00 \mathrm{E}-10$ & $1.00 \mathrm{E}-10$ & -26038.57 & -32667.41 \\
\hline ATO & 1.64 & 1.00 & $1.00 \mathrm{E}-10$ & $1.00 \mathrm{E}-10$ & -25284.84 & -15000.00 \\
\hline ATS & 2.04 & 3.69 & $1.00 \mathrm{E}-10$ & $1.00 \mathrm{E}-10$ & -26205.05 & -26205.05 \\
\hline ATT & 3.52 & 2.24 & $1.00 \mathrm{E}-10$ & $1.00 \mathrm{E}-10$ & -30535.31 & -19470.37 \\
\hline ATV & 1.10 & 1.18 & $1.00 \mathrm{E}-10$ & $1.00 \mathrm{E}-10$ & -21464.70 & -27926.47 \\
\hline AWO & 1.49 & 1.74 & $1.00 \mathrm{E}-10$ & $1.00 \mathrm{E}-10$ & -33143.24 & -24410.79 \\
\hline AWW & 4.11 & 1.62 & $1.00 \mathrm{E}-10$ & $1.00 \mathrm{E}-10$ & -26209.72 & -37580.00 \\
\hline $\mathrm{BCT}$ & 1.00 & 1.00 & $1.00 \mathrm{E}-10$ & $1.00 \mathrm{E}-10$ & -15000.00 & -15000.00 \\
\hline BEA & 6.08 & 1.00 & $1.00 \mathrm{E}-10$ & $1.00 \mathrm{E}-10$ & -22645.95 & -22645.95 \\
\hline $\mathrm{BEC}$ & 6.08 & 1.19 & $1.00 \mathrm{E}-10$ & $1.00 \mathrm{E}-10$ & -22226.20 & -22226.20 \\
\hline BIK & 1.95 & 1.00 & $1.00 \mathrm{E}-10$ & $1.00 \mathrm{E}-10$ & -30236.15 & -30236.15 \\
\hline $\mathrm{BOF}$ & 2.83 & 1.00 & $1.00 \mathrm{E}-10$ & $1.00 \mathrm{E}-10$ & -27793.43 & -15000.00 \\
\hline $\mathrm{BOG}$ & 3.58 & 2.80 & $1.00 \mathrm{E}-10$ & $1.00 \mathrm{E}-10$ & -23268.32 & -23268.32 \\
\hline $\mathrm{BOZ}$ & 7.00 & 1.00 & $1.00 \mathrm{E}-10$ & $1.00 \mathrm{E}-10$ & -23984.50 & -29962.69 \\
\hline $\mathrm{BPH}$ & 1.26 & 6.99 & $1.00 \mathrm{E}-10$ & $1.00 \mathrm{E}-10$ & -24506.58 & -24506.58 \\
\hline BRE & 2.56 & 2.31 & $1.00 \mathrm{E}-10$ & $1.00 \mathrm{E}-10$ & -18465.26 & -29863.31 \\
\hline CAN & 4.44 & 1.00 & $1.00 \mathrm{E}-10$ & $1.00 \mathrm{E}-10$ & -27529.05 & -16621.12 \\
\hline CAS & 1.69 & 1.14 & $1.00 \mathrm{E}-10$ & $1.00 \mathrm{E}-10$ & -28807.02 & -28807.02 \\
\hline $\mathrm{CDO}$ & 1.07 & 3.41 & $1.00 \mathrm{E}-10$ & $1.00 \mathrm{E}-10$ & -17765.19 & -25618.18 \\
\hline CFI & 4.05 & 1.00 & $1.00 \mathrm{E}-10$ & $1.00 \mathrm{E}-10$ & -22159.67 & -22159.67 \\
\hline CGF & 1.92 & 1.80 & $1.00 \mathrm{E}-10$ & $1.00 \mathrm{E}-10$ & -28863.78 & -16076.54 \\
\hline CGS & 3.63 & 1.11 & $1.00 \mathrm{E}-10$ & $1.00 \mathrm{E}-10$ & -26155.50 & -26155.50 \\
\hline CHA & 3.88 & 3.48 & $1.00 \mathrm{E}-10$ & $1.00 \mathrm{E}-10$ & -25168.60 & -25168.60 \\
\hline $\mathrm{CZP}$ & 1.00 & 1.66 & $1.00 \mathrm{E}-10$ & $1.00 \mathrm{E}-10$ & -18946.74 & -28937.33 \\
\hline $\mathrm{DAC}$ & 1.99 & 2.58 & $1.00 \mathrm{E}-10$ & $1.00 \mathrm{E}-10$ & -33018.10 & -26437.66 \\
\hline DDR & 1.00 & 3.68 & $1.00 \mathrm{E}-10$ & $1.00 \mathrm{E}-10$ & -26089.38 & -26089.38 \\
\hline DFO & 7.00 & 7.00 & $1.00 \mathrm{E}-10$ & $1.00 \mathrm{E}-10$ & -21766.37 & -21766.37 \\
\hline DFT & 1.00 & 3.99 & $1.00 \mathrm{E}-10$ & $1.00 \mathrm{E}-10$ & -15000.00 & -30672.49 \\
\hline $\mathrm{DOH}$ & 1.00 & 3.05 & $1.00 \mathrm{E}-10$ & $1.00 \mathrm{E}-10$ & -29738.55 & -26481.84 \\
\hline $\mathrm{DON}$ & 3.42 & 7.00 & $1.00 \mathrm{E}-10$ & $1.00 \mathrm{E}-10$ & -20654.92 & -15000.00 \\
\hline EAB & 4.57 & 1.00 & $1.00 \mathrm{E}-10$ & $1.00 \mathrm{E}-10$ & -26136.44 & -30254.83 \\
\hline EDI & 4.38 & 1.00 & $1.00 \mathrm{E}-10$ & $1.00 \mathrm{E}-10$ & -26117.83 & -15701.67 \\
\hline EMT & 7.00 & 7.00 & $1.00 \mathrm{E}-10$ & $1.00 \mathrm{E}-10$ & -21236.24 & -21236.24 \\
\hline EON & 1.00 & 3.94 & $1.00 \mathrm{E}-10$ & $1.00 \mathrm{E}-10$ & -32684.25 & -24712.23 \\
\hline EPI & 1.65 & 2.54 & $1.00 \mathrm{E}-10$ & $1.00 \mathrm{E}-10$ & -25194.03 & -30962.78 \\
\hline ERI & 3.06 & 1.99 & $1.00 \mathrm{E}-10$ & $1.00 \mathrm{E}-10$ & -25510.14 & -25510.14 \\
\hline ESV & 1.42 & 4.34 & $1.00 \mathrm{E}-10$ & $1.00 \mathrm{E}-10$ & -20584.78 & -28341.55 \\
\hline ETR & 5.35 & 3.01 & $1.00 \mathrm{E}-10$ & $1.00 \mathrm{E}-10$ & -23880.54 & -15000.00 \\
\hline EUO & 2.65 & 1.00 & $1.00 \mathrm{E}-10$ & $1.00 \mathrm{E}-10$ & -24914.84 & -24914.84 \\
\hline $\mathrm{EZT}$ & 1.00 & 2.94 & $1.00 \mathrm{E}-10$ & $1.00 \mathrm{E}-10$ & -24515.63 & -24515.63 \\
\hline FAR & 1.00 & 3.99 & $1.00 \mathrm{E}-10$ & $1.00 \mathrm{E}-10$ & -15000.00 & -28215.66 \\
\hline FAU & 7.00 & 7.00 & $1.00 \mathrm{E}-10$ & $1.00 \mathrm{E}-10$ & -21119.92 & -21119.92 \\
\hline FER & 2.73 & 1.00 & $1.00 \mathrm{E}-10$ & $1.00 \mathrm{E}-10$ & -26177.86 & -26177.86 \\
\hline FRA & 2.63 & 1.77 & $1.00 \mathrm{E}-10$ & $1.00 \mathrm{E}-10$ & -24223.14 & -29254.54 \\
\hline GIS & 4.94 & 3.99 & $1.00 \mathrm{E}-10$ & $1.00 \mathrm{E}-10$ & -31604.15 & -17501.26 \\
\hline GIU & 3.35 & 1.06 & $1.00 \mathrm{E}-10$ & $1.00 \mathrm{E}-10$ & -27313.29 & -27313.29 \\
\hline GME & 1.00 & 6.04 & $1.00 \mathrm{E}-10$ & $1.00 \mathrm{E}-10$ & -31668.17 & -25145.29 \\
\hline
\end{tabular}




\begin{tabular}{|c|c|c|c|c|c|c|}
\hline GON & 1.16 & 1.15 & $1.00 \mathrm{E}-10$ & $1.00 \mathrm{E}-10$ & -23717.81 & -23717.81 \\
\hline GOO & 2.31 & 2.01 & $1.00 \mathrm{E}-10$ & $1.00 \mathrm{E}-10$ & -28483.28 & -17690.29 \\
\hline $\mathrm{HEU}$ & 1.77 & 3.49 & $1.00 \mathrm{E}-10$ & $1.00 \mathrm{E}-10$ & -16949.02 & -29766.27 \\
\hline IFR & 1.00 & 3.77 & $1.00 \mathrm{E}-10$ & $1.00 \mathrm{E}-10$ & -23958.26 & -23958.26 \\
\hline IHW & 1.52 & 1.62 & $1.00 \mathrm{E}-10$ & $1.00 \mathrm{E}-10$ & -25900.63 & -25900.63 \\
\hline $\mathrm{IMF}$ & 7.00 & 7.00 & $1.00 \mathrm{E}-10$ & $1.00 \mathrm{E}-10$ & -22212.69 & -22212.69 \\
\hline IRR & 7.00 & 7.00 & $1.00 \mathrm{E}-10$ & $1.00 \mathrm{E}-10$ & -19779.76 & -19779.76 \\
\hline ISV & 5.08 & 2.62 & $1.00 \mathrm{E}-10$ & $1.00 \mathrm{E}-10$ & -22317.89 & -22317.89 \\
\hline ITE & 5.59 & 1.00 & $1.00 \mathrm{E}-10$ & $1.00 \mathrm{E}-10$ & -24467.23 & -24467.23 \\
\hline ITH & 1.83 & 2.24 & $1.00 \mathrm{E}-10$ & $1.00 \mathrm{E}-10$ & -25414.25 & -25414.25 \\
\hline ITR & 3.10 & 1.27 & $1.00 \mathrm{E}-10$ & $1.00 \mathrm{E}-10$ & -25328.35 & -25328.35 \\
\hline ITW & 1.76 & 3.64 & $1.00 \mathrm{E}-10$ & $1.00 \mathrm{E}-10$ & -18078.58 & -28989.84 \\
\hline IWR & 1.79 & 4.85 & $1.00 \mathrm{E}-10$ & $1.00 \mathrm{E}-10$ & -23598.06 & -23598.06 \\
\hline IWS & 7.00 & 7.00 & $1.00 \mathrm{E}-10$ & $1.00 \mathrm{E}-10$ & -20370.12 & -20370.12 \\
\hline IWV & 7.00 & 7.00 & $1.00 \mathrm{E}-10$ & $1.00 \mathrm{E}-10$ & -20729.92 & -20729.92 \\
\hline IWW & 4.46 & 1.00 & $1.00 \mathrm{E}-10$ & $1.00 \mathrm{E}-10$ & -23854.20 & -23854.20 \\
\hline JBW & 1.00 & 1.79 & $1.00 \mathrm{E}-10$ & $1.00 \mathrm{E}-10$ & -33239.63 & -33239.63 \\
\hline JOZ & 4.51 & 1.00 & $1.00 \mathrm{E}-10$ & $1.00 \mathrm{E}-10$ & -26796.74 & -16863.93 \\
\hline JRY & 1.00 & 2.96 & $1.00 \mathrm{E}-10$ & $1.00 \mathrm{E}-10$ & -16238.92 & -28285.08 \\
\hline JSN & 2.31 & 2.87 & $1.00 \mathrm{E}-10$ & $1.00 \mathrm{E}-10$ & -16762.69 & -27145.33 \\
\hline JST & 7.00 & 4.95 & $1.00 \mathrm{E}-10$ & $1.00 \mathrm{E}-10$ & -25153.79 & -25153.79 \\
\hline JSW & 1.71 & 1.23 & $1.00 \mathrm{E}-10$ & $1.00 \mathrm{E}-10$ & -29863.90 & -24106.24 \\
\hline KFI & 6.82 & 1.16 & $1.00 \mathrm{E}-10$ & $1.00 \mathrm{E}-10$ & -27039.18 & -16955.55 \\
\hline $\mathrm{LAU}$ & 1.87 & 3.09 & $1.00 \mathrm{E}-10$ & $1.00 \mathrm{E}-10$ & -15000.00 & -26452.80 \\
\hline LEV & 3.89 & 2.13 & $1.00 \mathrm{E}-10$ & $1.00 \mathrm{E}-10$ & -26217.48 & -26217.48 \\
\hline LIO & 1.12 & 3.37 & $1.00 \mathrm{E}-10$ & $1.00 \mathrm{E}-10$ & -20375.18 & -28166.61 \\
\hline LOS & 2.83 & 1.74 & $1.00 \mathrm{E}-10$ & $1.00 \mathrm{E}-10$ & -26138.60 & -36232.75 \\
\hline LOV & 5.09 & 1.00 & $1.00 \mathrm{E}-10$ & $1.00 \mathrm{E}-10$ & -27803.91 & -27803.91 \\
\hline LTA & 1.98 & 7.00 & $1.00 \mathrm{E}-10$ & $1.00 \mathrm{E}-10$ & -23425.92 & -23425.92 \\
\hline LTF & 6.61 & 3.53 & $1.00 \mathrm{E}-10$ & $1.00 \mathrm{E}-10$ & -15000.00 & -27337.50 \\
\hline LTJ & 1.39 & 2.94 & $1.00 \mathrm{E}-10$ & $1.00 \mathrm{E}-10$ & -22145.43 & -22145.43 \\
\hline LTL & 3.04 & 1.08 & $1.00 \mathrm{E}-10$ & $1.00 \mathrm{E}-10$ & -25328.09 & -25328.09 \\
\hline LTN & 4.37 & 1.00 & $1.00 \mathrm{E}-10$ & $1.00 \mathrm{E}-10$ & -25629.32 & -29053.43 \\
\hline MAR & 1.26 & 2.29 & $1.00 \mathrm{E}-10$ & $1.00 \mathrm{E}-10$ & -36320.85 & -27706.96 \\
\hline MAZ & 4.36 & 1.00 & $1.00 \mathrm{E}-10$ & $1.00 \mathrm{E}-10$ & -26751.97 & -19282.77 \\
\hline MEI & 2.94 & 6.62 & $1.00 \mathrm{E}-10$ & $1.00 \mathrm{E}-10$ & -22730.90 & -22730.90 \\
\hline MEL & 2.81 & 1.00 & $1.00 \mathrm{E}-10$ & $1.00 \mathrm{E}-10$ & -25733.86 & -25733.86 \\
\hline MEP & 2.03 & 2.81 & $1.00 \mathrm{E}-10$ & $1.00 \mathrm{E}-10$ & -15000.00 & -29561.86 \\
\hline MER & 1.47 & 5.10 & $1.00 \mathrm{E}-10$ & $1.00 \mathrm{E}-10$ & -18485.67 & -29049.54 \\
\hline MFI & 2.36 & 1.00 & $1.00 \mathrm{E}-10$ & $1.00 \mathrm{E}-10$ & -26996.54 & -26996.54 \\
\hline MFS & 1.34 & 2.62 & $1.00 \mathrm{E}-10$ & $1.00 \mathrm{E}-10$ & -26527.87 & -26527.87 \\
\hline MON & 6.26 & 1.00 & $1.00 \mathrm{E}-10$ & $1.00 \mathrm{E}-10$ & -28324.59 & -28324.59 \\
\hline MOR & 1.48 & 3.31 & $1.00 \mathrm{E}-10$ & $1.00 \mathrm{E}-10$ & -32199.52 & -23621.21 \\
\hline MOZ & 1.00 & 7.00 & $1.00 \mathrm{E}-10$ & $1.00 \mathrm{E}-10$ & -29087.39 & -22788.12 \\
\hline MRE & 1.00 & 1.36 & $1.00 \mathrm{E}-10$ & $1.00 \mathrm{E}-10$ & -15000.00 & -26438.17 \\
\hline MSE & 3.63 & 1.20 & $1.00 \mathrm{E}-10$ & $1.00 \mathrm{E}-10$ & -24456.48 & -24456.48 \\
\hline MSO & 2.04 & 1.00 & $1.00 \mathrm{E}-10$ & $1.00 \mathrm{E}-10$ & -25282.04 & -25282.04 \\
\hline
\end{tabular}




\begin{tabular}{|c|c|c|c|c|c|c|}
\hline MTF & 1.43 & 1.81 & $1.00 \mathrm{E}-10$ & $1.00 \mathrm{E}-10$ & -16038.63 & -27748.60 \\
\hline MTN & 2.85 & 1.41 & $1.00 \mathrm{E}-10$ & $1.00 \mathrm{E}-10$ & -26315.57 & -26315.57 \\
\hline MTT & 2.61 & 1.64 & $1.00 \mathrm{E}-10$ & $1.00 \mathrm{E}-10$ & -27532.88 & -17380.58 \\
\hline MTW & 1.88 & 1.07 & $1.00 \mathrm{E}-10$ & $1.00 \mathrm{E}-10$ & -27776.30 & -27776.30 \\
\hline MVY & 1.00 & 1.82 & $1.00 \mathrm{E}-10$ & $1.00 \mathrm{E}-10$ & -28795.27 & -28795.27 \\
\hline MWW & 5.06 & 2.36 & $1.00 \mathrm{E}-10$ & $1.00 \mathrm{E}-10$ & -25138.80 & -15000.00 \\
\hline NAB & 1.80 & 7.00 & $1.00 \mathrm{E}-10$ & $1.00 \mathrm{E}-10$ & -24847.80 & -24847.80 \\
\hline NAT & 3.17 & 1.82 & $1.00 \mathrm{E}-10$ & $1.00 \mathrm{E}-10$ & -25174.43 & -25174.43 \\
\hline NES & 4.31 & 1.00 & $1.00 \mathrm{E}-10$ & $1.00 \mathrm{E}-10$ & -24245.16 & -24245.16 \\
\hline $\mathrm{NON}$ & 2.17 & 2.46 & $1.00 \mathrm{E}-10$ & $1.00 \mathrm{E}-10$ & -25952.98 & -15000.00 \\
\hline NPO & 1.00 & 1.00 & $1.00 \mathrm{E}-10$ & $1.00 \mathrm{E}-10$ & -19809.27 & -24619.67 \\
\hline NPT & 7.00 & 3.29 & $1.00 \mathrm{E}-10$ & $1.00 \mathrm{E}-10$ & -25023.97 & -25023.97 \\
\hline NSI & 1.46 & 1.36 & $1.00 \mathrm{E}-10$ & $1.00 \mathrm{E}-10$ & -29041.71 & -29041.71 \\
\hline OBW & 6.05 & 7.00 & $1.00 \mathrm{E}-10$ & $1.00 \mathrm{E}-10$ & -22957.23 & -22957.23 \\
\hline $\mathrm{OFF}$ & 1.00 & 4.02 & $1.00 \mathrm{E}-10$ & $1.00 \mathrm{E}-10$ & -29704.36 & -24756.34 \\
\hline OSI & 3.05 & 1.00 & $1.00 \mathrm{E}-10$ & $1.00 \mathrm{E}-10$ & -23521.33 & -23521.33 \\
\hline OSO & 7.00 & 4.09 & $1.00 \mathrm{E}-10$ & $1.00 \mathrm{E}-10$ & -22838.78 & -22838.78 \\
\hline OWE & 4.22 & 1.55 & $1.00 \mathrm{E}-10$ & $1.00 \mathrm{E}-10$ & -28074.73 & -17523.46 \\
\hline $\mathrm{PAU}$ & 2.72 & 2.72 & $1.00 \mathrm{E}-10$ & $1.00 \mathrm{E}-10$ & -28693.57 & -28693.57 \\
\hline PCR & 2.45 & 2.42 & $1.00 \mathrm{E}-10$ & $1.00 \mathrm{E}-10$ & -15000.00 & -27101.20 \\
\hline PHI & 1.89 & 5.34 & $1.00 \mathrm{E}-10$ & $1.00 \mathrm{E}-10$ & -17794.89 & -29395.63 \\
\hline $\mathrm{PON}$ & 2.24 & 1.02 & $1.00 \mathrm{E}-10$ & $1.00 \mathrm{E}-10$ & -27259.72 & -27259.72 \\
\hline PUN & 1.00 & 6.96 & $1.00 \mathrm{E}-10$ & $1.00 \mathrm{E}-10$ & -27127.72 & -27127.72 \\
\hline RHO & 1.93 & 6.03 & $1.00 \mathrm{E}-10$ & $1.00 \mathrm{E}-10$ & -31002.33 & -24794.79 \\
\hline $\mathrm{RRO}$ & 2.08 & 1.93 & $1.00 \mathrm{E}-10$ & $1.00 \mathrm{E}-10$ & -30108.65 & -30108.65 \\
\hline $\mathrm{RSN}$ & 3.64 & 3.20 & $1.00 \mathrm{E}-10$ & $1.00 \mathrm{E}-10$ & -26689.35 & -26689.35 \\
\hline RTE & 3.50 & 1.36 & $1.00 \mathrm{E}-10$ & $1.00 \mathrm{E}-10$ & -26651.62 & -26651.62 \\
\hline $\mathrm{RTH}$ & 2.71 & 4.08 & $1.00 \mathrm{E}-10$ & $1.00 \mathrm{E}-10$ & -24499.90 & -24499.90 \\
\hline RUT & 3.03 & 2.39 & $1.00 \mathrm{E}-10$ & $1.00 \mathrm{E}-10$ & -19488.72 & -29403.47 \\
\hline RWR & 3.00 & 1.00 & $1.00 \mathrm{E}-10$ & $1.00 \mathrm{E}-10$ & -29813.99 & -29813.99 \\
\hline RWY & 7.00 & 7.00 & $1.00 \mathrm{E}-10$ & $1.00 \mathrm{E}-10$ & -20069.15 & -20069.15 \\
\hline $\mathrm{SAF}$ & 1.67 & 1.00 & $1.00 \mathrm{E}-10$ & $1.00 \mathrm{E}-10$ & -22833.23 & -22833.23 \\
\hline $\mathrm{SAO}$ & 5.53 & 5.56 & $1.00 \mathrm{E}-10$ & $1.00 \mathrm{E}-10$ & -20994.30 & -20994.30 \\
\hline SAS & 1.14 & 4.94 & $1.00 \mathrm{E}-10$ & $1.00 \mathrm{E}-10$ & -24247.18 & -24247.18 \\
\hline SAT & 3.14 & 1.00 & $1.00 \mathrm{E}-10$ & $1.00 \mathrm{E}-10$ & -25284.25 & -25284.25 \\
\hline SAV & 3.95 & 4.06 & $1.00 \mathrm{E}-10$ & $1.00 \mathrm{E}-10$ & -25654.16 & -25654.16 \\
\hline SBE & 1.00 & 7.00 & $1.00 \mathrm{E}-10$ & $1.00 \mathrm{E}-10$ & -28865.73 & -22123.30 \\
\hline SBN & 4.85 & 1.37 & $1.00 \mathrm{E}-10$ & $1.00 \mathrm{E}-10$ & -23808.44 & -23808.44 \\
\hline SBS & 7.00 & 7.00 & $1.00 \mathrm{E}-10$ & $1.00 \mathrm{E}-10$ & -19812.77 & -19812.77 \\
\hline SBT & 7.00 & 7.00 & $1.00 \mathrm{E}-10$ & $1.00 \mathrm{E}-10$ & -19703.46 & -19703.46 \\
\hline SFE & 3.65 & 1.39 & $1.00 \mathrm{E}-10$ & $1.00 \mathrm{E}-10$ & -25637.00 & -25637.00 \\
\hline SFF & 1.95 & 2.70 & $1.00 \mathrm{E}-10$ & $1.00 \mathrm{E}-10$ & -24769.79 & -24769.79 \\
\hline SFG & 1.29 & 1.70 & $1.00 \mathrm{E}-10$ & $1.00 \mathrm{E}-10$ & -25008.95 & -25008.95 \\
\hline SFH & 1.00 & 4.98 & $1.00 \mathrm{E}-10$ & $1.00 \mathrm{E}-10$ & -24203.55 & -24203.56 \\
\hline SFN & 3.42 & 1.90 & $1.00 \mathrm{E}-10$ & $1.00 \mathrm{E}-10$ & -24536.01 & -24536.01 \\
\hline $\mathrm{SFO}$ & 4.45 & 3.09 & $1.00 \mathrm{E}-10$ & $1.00 \mathrm{E}-10$ & -23598.47 & -23598.47 \\
\hline SFS & 1.00 & 3.91 & $1.00 \mathrm{E}-10$ & $1.00 \mathrm{E}-10$ & -28424.92 & -23707.89 \\
\hline
\end{tabular}




\begin{tabular}{|c|c|c|c|c|c|c|}
\hline SFV & 1.78 & 3.03 & $1.00 \mathrm{E}-10$ & $1.00 \mathrm{E}-10$ & -15455.38 & -27291.53 \\
\hline SGT & 3.17 & 3.02 & $1.00 \mathrm{E}-10$ & $1.00 \mathrm{E}-10$ & -15000.00 & -25448.47 \\
\hline SIV & 5.00 & 3.01 & $1.00 \mathrm{E}-10$ & $1.00 \mathrm{E}-10$ & -30722.26 & -18056.37 \\
\hline SOD & 3.59 & 3.13 & $1.00 \mathrm{E}-10$ & $1.00 \mathrm{E}-10$ & -17484.33 & -29444.29 \\
\hline SOF & 1.00 & 4.70 & $1.00 \mathrm{E}-10$ & $1.00 \mathrm{E}-10$ & -26751.22 & -26751.22 \\
\hline SOS & 1.00 & 4.41 & $1.00 \mathrm{E}-10$ & $1.00 \mathrm{E}-10$ & -24827.77 & -24827.77 \\
\hline $\mathrm{SSF}$ & 3.90 & 1.00 & $1.00 \mathrm{E}-10$ & $1.00 \mathrm{E}-10$ & -22260.63 & -22260.63 \\
\hline SSY & 3.64 & 1.38 & $1.00 \mathrm{E}-10$ & $1.00 \mathrm{E}-10$ & -25644.89 & -25644.89 \\
\hline STF & 4.87 & 1.00 & $1.00 \mathrm{E}-10$ & $1.00 \mathrm{E}-10$ & -24555.73 & -24555.73 \\
\hline STI & 1.59 & 3.49 & $1.00 \mathrm{E}-10$ & $1.00 \mathrm{E}-10$ & -26030.84 & -26030.84 \\
\hline STO & 1.10 & 1.67 & $1.00 \mathrm{E}-10$ & $1.00 \mathrm{E}-10$ & -22908.08 & -22908.08 \\
\hline STT & 1.94 & 2.65 & $1.00 \mathrm{E}-10$ & $1.00 \mathrm{E}-10$ & -26215.37 & -26215.37 \\
\hline STW & 2.51 & 2.91 & $1.00 \mathrm{E}-10$ & $1.00 \mathrm{E}-10$ & -27957.49 & -27957.49 \\
\hline SZR & 2.32 & 1.85 & $1.00 \mathrm{E}-10$ & $1.00 \mathrm{E}-10$ & -26731.52 & -26731.52 \\
\hline TER & 1.00 & 3.04 & $1.00 \mathrm{E}-10$ & $1.00 \mathrm{E}-10$ & -26511.43 & -26511.43 \\
\hline THO & 3.01 & 2.02 & $1.00 \mathrm{E}-10$ & $1.00 \mathrm{E}-10$ & -25092.04 & -25092.04 \\
\hline TOL & 2.79 & 1.00 & $1.00 \mathrm{E}-10$ & $1.00 \mathrm{E}-10$ & -25844.21 & -36300.60 \\
\hline TON & 1.13 & 1.81 & $1.00 \mathrm{E}-10$ & $1.00 \mathrm{E}-10$ & -27045.14 & -27045.14 \\
\hline TSC & 1.00 & 1.06 & $1.00 \mathrm{E}-10$ & $1.00 \mathrm{E}-10$ & -15000.00 & -32364.59 \\
\hline TUN & 7.00 & 7.00 & $1.00 \mathrm{E}-10$ & $1.00 \mathrm{E}-10$ & -20075.23 & -23637.49 \\
\hline UEI & 1.93 & 2.12 & $1.00 \mathrm{E}-10$ & $1.00 \mathrm{E}-10$ & -31445.55 & -23873.25 \\
\hline UFI & 1.00 & 5.94 & $1.00 \mathrm{E}-10$ & $1.00 \mathrm{E}-10$ & -30767.66 & -24488.59 \\
\hline UOS & 3.24 & 1.00 & $1.00 \mathrm{E}-10$ & $1.00 \mathrm{E}-10$ & -25771.75 & -15000.00 \\
\hline UOZ & 1.64 & 1.00 & $1.00 \mathrm{E}-10$ & $1.00 \mathrm{E}-10$ & -29523.79 & -29523.79 \\
\hline USI & 2.84 & 2.77 & $1.00 \mathrm{E}-10$ & $1.00 \mathrm{E}-10$ & -24818.05 & -24818.05 \\
\hline UTL & 1.00 & 5.82 & $1.00 \mathrm{E}-10$ & $1.00 \mathrm{E}-10$ & -22805.39 & -22805.39 \\
\hline UWY & 7.00 & 5.61 & $1.00 \mathrm{E}-10$ & $1.00 \mathrm{E}-10$ & -15000.00 & -23828.92 \\
\hline VET & 2.12 & 2.09 & $1.00 \mathrm{E}-10$ & $1.00 \mathrm{E}-10$ & -15000.00 & -24196.15 \\
\hline VFI & 7.00 & 7.00 & $1.00 \mathrm{E}-10$ & $1.00 \mathrm{E}-10$ & -18529.58 & -18529.58 \\
\hline VNI & 3.15 & 1.09 & $1.00 \mathrm{E}-10$ & $1.00 \mathrm{E}-10$ & -23921.09 & -23921.09 \\
\hline VSV & 7.00 & 1.22 & $1.00 \mathrm{E}-10$ & $1.00 \mathrm{E}-10$ & -24873.27 & -24873.27 \\
\hline WEI & 1.00 & 6.63 & $1.00 \mathrm{E}-10$ & $1.00 \mathrm{E}-10$ & -30670.54 & -30670.54 \\
\hline YUG & 3.67 & 1.00 & $1.00 \mathrm{E}-10$ & $1.00 \mathrm{E}-10$ & -29345.58 & -29345.58 \\
\hline $\mathrm{ZON}$ & 1.78 & 3.03 & $1.00 \mathrm{E}-10$ & $1.00 \mathrm{E}-10$ & -15455.38 & -27291.53 \\
\hline
\end{tabular}

Table S3: Fitted dual-site adsorption isotherm parameters for $\mathrm{CO}_{2}$ adsorption on zeolites.

\section{S5.2 $\mathrm{N}_{2}$ adsorption isotherm parameters on single-site model}

\begin{tabular}{cccc}
\hline Adsorbent & $\begin{array}{c}m_{2,1} \\
\left(\mathrm{~mol} \mathrm{~kg}^{-1}\right)\end{array}$ & $\begin{array}{c}b_{2,1} \\
\left(\mathrm{~Pa}^{-1}\right)\end{array}$ & $\begin{array}{c}\Delta U_{2,1} \\
\left(\mathrm{~kJ} \mathrm{~mol}^{-1}\right)\end{array}$ \\
\hline ABW & 1.50 & $1.00 \mathrm{E}-10$ & -20812.27 \\
ACO & 1.50 & $1.00 \mathrm{E}-10$ & -21541.55 \\
AEI & 1.50 & $1.00 \mathrm{E}-10$ & -22574.57 \\
AEL & 1.50 & $1.00 \mathrm{E}-10$ & -21165.78 \\
AEN & 1.50 & $1.00 \mathrm{E}-10$ & -19998.09
\end{tabular}




\begin{tabular}{|c|c|c|c|}
\hline AET & 1.50 & $1.00 \mathrm{E}-10$ & -19343.05 \\
\hline $\mathrm{AFG}$ & 1.50 & $1.00 \mathrm{E}-10$ & -22592.33 \\
\hline AFI & 1.50 & $1.00 \mathrm{E}-10$ & -21466.87 \\
\hline $\mathrm{AFN}$ & 1.50 & $1.00 \mathrm{E}-10$ & -21457.48 \\
\hline $\mathrm{AFO}$ & 1.50 & $1.00 \mathrm{E}-10$ & -21083.60 \\
\hline AFR & 1.50 & $1.00 \mathrm{E}-10$ & -21912.76 \\
\hline AFS & 1.50 & $1.00 \mathrm{E}-10$ & -21904.99 \\
\hline $\mathrm{AFT}$ & 1.50 & $1.00 \mathrm{E}-10$ & -22553.99 \\
\hline AFX & 1.50 & $1.00 \mathrm{E}-10$ & -22542.92 \\
\hline $\mathrm{AFY}$ & 1.50 & $1.00 \mathrm{E}-10$ & -22734.10 \\
\hline AHT & 1.50 & $1.00 \mathrm{E}-10$ & -16650.36 \\
\hline ANA & 1.50 & $1.00 \mathrm{E}-10$ & -17633.35 \\
\hline $\mathrm{APC}$ & 1.50 & $1.00 \mathrm{E}-10$ & -20683.37 \\
\hline $\mathrm{APD}$ & 1.50 & $1.00 \mathrm{E}-10$ & -22599.49 \\
\hline $\mathrm{AST}$ & 1.50 & $1.00 \mathrm{E}-10$ & -23236.45 \\
\hline $\mathrm{ASV}$ & 1.50 & $1.00 \mathrm{E}-10$ & -22016.13 \\
\hline ATN & 1.50 & $1.00 \mathrm{E}-10$ & -22849.22 \\
\hline ATO & 1.50 & $1.00 \mathrm{E}-10$ & -20588.45 \\
\hline ATS & 1.50 & $1.00 \mathrm{E}-10$ & -21468.03 \\
\hline ATT & 1.50 & $1.00 \mathrm{E}-10$ & -22929.57 \\
\hline ATV & 1.50 & $1.00 \mathrm{E}-10$ & -21063.45 \\
\hline AWO & 1.50 & $1.00 \mathrm{E}-10$ & -21792.94 \\
\hline AWW & 1.50 & $1.00 \mathrm{E}-10$ & -22552.59 \\
\hline $\mathrm{BCT}$ & 1.50 & $1.00 \mathrm{E}-10$ & -20652.75 \\
\hline BEA & 1.50 & $1.00 \mathrm{E}-10$ & -22016.33 \\
\hline BEC & 1.50 & $1.00 \mathrm{E}-10$ & -22006.56 \\
\hline BIK & 1.50 & $1.00 \mathrm{E}-10$ & -20036.24 \\
\hline $\mathrm{BOF}$ & 1.50 & $1.00 \mathrm{E}-10$ & -22557.76 \\
\hline $\mathrm{BOG}$ & 1.50 & $1.00 \mathrm{E}-10$ & -21823.14 \\
\hline BOZ & 1.50 & $1.00 \mathrm{E}-10$ & -22254.26 \\
\hline BPH & 1.50 & $1.00 \mathrm{E}-10$ & -21922.75 \\
\hline BRE & 1.50 & $1.00 \mathrm{E}-10$ & -22461.52 \\
\hline $\mathrm{CAN}$ & 1.50 & $1.00 \mathrm{E}-10$ & -22494.01 \\
\hline CAS & 1.50 & $1.00 \mathrm{E}-10$ & -22138.90 \\
\hline $\mathrm{CDO}$ & 1.50 & $1.00 \mathrm{E}-10$ & -21797.49 \\
\hline CFI & 1.50 & $1.00 \mathrm{E}-10$ & -20277.01 \\
\hline CGF & 1.50 & $1.00 \mathrm{E}-10$ & -22278.51 \\
\hline CGS & 1.50 & $1.00 \mathrm{E}-10$ & -22124.23 \\
\hline $\mathrm{CHA}$ & 1.50 & $1.00 \mathrm{E}-10$ & -22616.76 \\
\hline $\mathrm{CZP}$ & 1.50 & $1.00 \mathrm{E}-10$ & -20742.80 \\
\hline DAC & 1.50 & $1.00 \mathrm{E}-10$ & -23283.15 \\
\hline DDR & 1.50 & $1.00 \mathrm{E}-10$ & -23453.91 \\
\hline DFO & 1.50 & $1.00 \mathrm{E}-10$ & -22053.90 \\
\hline DFT & 1.50 & $1.00 \mathrm{E}-10$ & -23135.49 \\
\hline $\mathrm{DOH}$ & 1.50 & $1.00 \mathrm{E}-10$ & -24703.07 \\
\hline $\mathrm{DON}$ & 1.50 & $1.00 \mathrm{E}-10$ & -19910.66 \\
\hline EAB & 1.50 & $1.00 \mathrm{E}-10$ & -23192.38 \\
\hline
\end{tabular}




$\begin{array}{clll}\text { EDI } & 1.50 & 1.00 \mathrm{E}-10 & -22616.03 \\ \text { EMT } & 1.50 & 1.00 \mathrm{E}-10 & -22691.53 \\ \text { EON } & 1.50 & 1.00 \mathrm{E}-10 & -22439.09 \\ \text { EPI } & 1.50 & 1.00 \mathrm{E}-10 & -23086.35 \\ \text { ERI } & 1.50 & 1.00 \mathrm{E}-10 & -22369.96 \\ \text { ESV } & 1.50 & 1.00 \mathrm{E}-10 & -22942.14 \\ \text { ETR } & 1.50 & 1.00 \mathrm{E}-10 & -21820.62 \\ \text { EUO } & 1.50 & 1.00 \mathrm{E}-10 & -21508.95 \\ \text { EZT } & 1.50 & 1.00 \mathrm{E}-10 & -21848.84 \\ \text { FAR } & 1.50 & 1.00 \mathrm{E}-10 & -23307.16 \\ \text { FAU } & 1.50 & 1.00 \mathrm{E}-10 & -22595.49 \\ \text { FER } & 1.50 & 1.00 \mathrm{E}-10 & -22641.25 \\ \text { FRA } & 1.50 & 1.00 \mathrm{E}-10 & -23535.80 \\ \text { GIS } & 1.50 & 1.00 \mathrm{E}-10 & -22829.65 \\ \text { GIU } & 1.50 & 1.00 \mathrm{E}-10 & -22853.08 \\ \text { GME } & 1.50 & 1.00 \mathrm{E}-10 & -22524.54 \\ \text { GON } & 1.50 & 1.00 \mathrm{E}-10 & -20450.14 \\ \text { GOO } & 1.50 & 1.00 \mathrm{E}-10 & -20772.33 \\ \text { HEU } & 1.50 & 1.00 \mathrm{E}-10 & -22766.25 \\ \text { IFR } & 1.50 & 1.00 \mathrm{E}-10 & -22019.31 \\ \text { IHW } & 1.50 & 1.00 \mathrm{E}-10 & -21508.96 \\ \text { IMF } & 1.50 & 1.00 \mathrm{E}-10 & -22024.13 \\ \text { IRR } & 1.50 & 1.00 \mathrm{E}-10 & -21613.44 \\ \text { ISV } & 1.50 & 1.00 \mathrm{E}-10 & -21907.35 \\ \text { ITE } & 1.50 & 1.00 \mathrm{E}-10 & -22258.20 \\ \text { ITH } & 1.50 & 1.00 \mathrm{E}-10 & -21831.04 \\ \text { ITR } & 1.50 & 1.00 \mathrm{E}-10 & -21845.41 \\ \text { ITW } & 1.50 & 1.00 \mathrm{E}-10 & -22404.83 \\ \text { IWR } & 1.50 & 1.00 \mathrm{E}-10 & -22127.32 \\ \text { IWS } & 1.50 & 1.00 \mathrm{E}-10 & -21701.14 \\ \text { IWV } & 1.50 & 1.00 \mathrm{E}-10 & -21332.36 \\ \text { IWW } & 1.50 & 1.00 \mathrm{E}-10 & -21743.53 \\ \text { JBW } & 1.50 & 1.00 \mathrm{E}-10 & -20826.34 \\ \text { JOZ } & 1.50 & 1.00 \mathrm{E}-10 & -21490.41 \\ \text { JRY } & 1.50 & 1.00 \mathrm{E}-10 & -22348.55 \\ \text { JSN } & 1.50 & 1.00 \mathrm{E}-10 & -22686.47 \\ \text { JST } & 1.50 & 1.00 \mathrm{E}-10 & -22599.33 \\ \text { JSW } & 1.50 & 1.00 \mathrm{E}-10 & -21961.84 \\ \text { KFI } & 1.50 & 1.00 \mathrm{E}-10 & -22398.82 \\ \text { LAU } & 1.50 & 1.00 \mathrm{E}-10 & -22245.87 \\ \text { LEV } & 1.50 & 1.00 \mathrm{E}-10 & -23015.72 \\ \text { LIO } & 1.50 & 1.00 \mathrm{E}-10 & -23392.69 \\ \text { LOS } & 1.50 & 1.00 \mathrm{E}-10 & -23317.61 \\ \text { LTA } & 1.50 & 1.00 \mathrm{E}-10 & -22623.95 \\ \text { LTF } & 1.50 & 1.00 \mathrm{E}-10 & -23047.79 \\ & 1.50 & 1.00 \mathrm{E}-10 & -22065.33 \\ \text { ITH } & & 1.00 \mathrm{E}-10 & -18864.45\end{array}$




\begin{tabular}{|c|c|c|c|}
\hline LTL & 1.50 & $1.00 \mathrm{E}-10$ & -20653.38 \\
\hline LTN & 1.50 & $1.00 \mathrm{E}-10$ & -22938.85 \\
\hline MAR & 1.50 & $1.00 \mathrm{E}-10$ & -23285.19 \\
\hline MAZ & 1.50 & $1.00 \mathrm{E}-10$ & -22196.07 \\
\hline MEI & 1.50 & $1.00 \mathrm{E}-10$ & -22084.81 \\
\hline MEL & 1.50 & $1.00 \mathrm{E}-10$ & -22079.75 \\
\hline MEP & 1.50 & $1.00 \mathrm{E}-10$ & -24646.51 \\
\hline MER & 1.50 & $1.00 \mathrm{E}-10$ & -22611.54 \\
\hline MFI & 1.50 & $1.00 \mathrm{E}-10$ & -22020.15 \\
\hline MFS & 1.50 & $1.00 \mathrm{E}-10$ & -21527.52 \\
\hline MON & 1.50 & $1.00 \mathrm{E}-10$ & -21252.16 \\
\hline MOR & 1.50 & $1.00 \mathrm{E}-10$ & -22637.27 \\
\hline MOZ & 1.50 & $1.00 \mathrm{E}-10$ & -21550.46 \\
\hline MRE & 1.50 & $1.00 \mathrm{E}-10$ & -20786.37 \\
\hline MSE & 1.50 & $1.00 \mathrm{E}-10$ & -21835.32 \\
\hline MSO & 1.50 & $1.00 \mathrm{E}-10$ & -21405.66 \\
\hline MTF & 1.50 & $1.00 \mathrm{E}-10$ & -21205.66 \\
\hline MTN & 1.50 & $1.00 \mathrm{E}-10$ & -24738.70 \\
\hline MTT & 1.50 & $1.00 \mathrm{E}-10$ & -21216.13 \\
\hline MTW & 1.50 & $1.00 \mathrm{E}-10$ & -20936.53 \\
\hline MVY & 0.92 & $1.00 \mathrm{E}-10$ & -10000.00 \\
\hline MWW & 1.50 & $1.00 \mathrm{E}-10$ & -22450.37 \\
\hline $\mathrm{NAB}$ & 1.50 & $1.00 \mathrm{E}-10$ & -19960.66 \\
\hline NAT & 1.50 & $1.00 \mathrm{E}-10$ & -22210.98 \\
\hline NES & 1.50 & $1.00 \mathrm{E}-10$ & -21656.01 \\
\hline $\mathrm{NON}$ & 1.50 & $1.00 \mathrm{E}-10$ & -21497.74 \\
\hline NPO & 1.50 & $1.00 \mathrm{E}-10$ & -17247.53 \\
\hline NPT & 1.50 & $1.00 \mathrm{E}-10$ & -22327.99 \\
\hline NSI & 1.50 & $1.00 \mathrm{E}-10$ & -18708.48 \\
\hline OBW & 1.50 & $1.00 \mathrm{E}-10$ & -22384.21 \\
\hline $\mathrm{OFF}$ & 1.50 & $1.00 \mathrm{E}-10$ & -22658.39 \\
\hline OSI & 1.50 & $1.00 \mathrm{E}-10$ & -20290.82 \\
\hline OSO & 1.50 & $1.00 \mathrm{E}-10$ & -22263.65 \\
\hline OWE & 1.50 & $1.00 \mathrm{E}-10$ & -22776.09 \\
\hline PAU & 1.50 & $1.00 \mathrm{E}-10$ & -22746.30 \\
\hline PCR & 1.50 & $1.00 \mathrm{E}-10$ & -21983.83 \\
\hline PHI & 1.50 & $1.00 \mathrm{E}-10$ & -22631.12 \\
\hline $\mathrm{PON}$ & 1.50 & $1.00 \mathrm{E}-10$ & -21989.24 \\
\hline PUN & 1.50 & $1.00 \mathrm{E}-10$ & -22819.28 \\
\hline RHO & 1.50 & $1.00 \mathrm{E}-10$ & -23344.33 \\
\hline $\mathrm{RRO}$ & 1.50 & $1.00 \mathrm{E}-10$ & -21240.91 \\
\hline $\mathrm{RSN}$ & 1.50 & $1.00 \mathrm{E}-10$ & -21821.62 \\
\hline RTE & 1.50 & $1.00 \mathrm{E}-10$ & -22615.59 \\
\hline RTH & 1.50 & $1.00 \mathrm{E}-10$ & -22301.29 \\
\hline RUT & 1.50 & $1.00 \mathrm{E}-10$ & -22969.29 \\
\hline RWR & 1.50 & $1.00 \mathrm{E}-10$ & -21474.93 \\
\hline RWY & 1.50 & $1.00 \mathrm{E}-10$ & -22448.78 \\
\hline
\end{tabular}




\begin{tabular}{|c|c|c|c|}
\hline SAF & 1.50 & $1.00 \mathrm{E}-10$ & -19891.91 \\
\hline $\mathrm{SAO}$ & 1.50 & $1.00 \mathrm{E}-10$ & -21933.74 \\
\hline SAS & 1.50 & $1.00 \mathrm{E}-10$ & -22183.25 \\
\hline SAT & 1.50 & $1.00 \mathrm{E}-10$ & -22072.06 \\
\hline SAV & 1.50 & $1.00 \mathrm{E}-10$ & -22387.83 \\
\hline SBE & 1.50 & $1.00 \mathrm{E}-10$ & -21934.31 \\
\hline SBN & 1.50 & $1.00 \mathrm{E}-10$ & -22585.44 \\
\hline SBS & 1.50 & $1.00 \mathrm{E}-10$ & -21471.62 \\
\hline SBT & 1.50 & $1.00 \mathrm{E}-10$ & -21453.99 \\
\hline SFE & 1.50 & $1.00 \mathrm{E}-10$ & -21016.46 \\
\hline SFF & 1.50 & $1.00 \mathrm{E}-10$ & -21830.76 \\
\hline SFG & 1.50 & $1.00 \mathrm{E}-10$ & -21145.73 \\
\hline SFH & 1.50 & $1.00 \mathrm{E}-10$ & -20593.02 \\
\hline SFN & 1.50 & $1.00 \mathrm{E}-10$ & -20608.80 \\
\hline SFO & 1.50 & $1.00 \mathrm{E}-10$ & -21820.87 \\
\hline SFS & 1.50 & $1.00 \mathrm{E}-10$ & -21926.89 \\
\hline SFV & 1.50 & $1.00 \mathrm{E}-10$ & -22720.44 \\
\hline SGT & 1.50 & $1.00 \mathrm{E}-10$ & -22484.69 \\
\hline SIV & 1.50 & $1.00 \mathrm{E}-10$ & -22785.26 \\
\hline SOD & 1.50 & $1.00 \mathrm{E}-10$ & -24269.51 \\
\hline $\mathrm{SOF}$ & 1.50 & $1.00 \mathrm{E}-10$ & -22745.26 \\
\hline SOS & 1.50 & $1.00 \mathrm{E}-10$ & -20681.72 \\
\hline SSF & 1.50 & $1.00 \mathrm{E}-10$ & -20838.03 \\
\hline SSY & 1.50 & $1.00 \mathrm{E}-10$ & -21004.94 \\
\hline STF & 1.50 & $1.00 \mathrm{E}-10$ & -22087.64 \\
\hline STI & 1.50 & $1.00 \mathrm{E}-10$ & -22550.28 \\
\hline STO & 1.50 & $1.00 \mathrm{E}-10$ & -20281.42 \\
\hline STT & 1.50 & $1.00 \mathrm{E}-10$ & -22300.49 \\
\hline STW & 1.50 & $1.00 \mathrm{E}-10$ & -23264.68 \\
\hline SZR & 1.50 & $1.00 \mathrm{E}-10$ & -21280.44 \\
\hline TER & 1.50 & $1.00 \mathrm{E}-10$ & -22428.54 \\
\hline THO & 1.50 & $1.00 \mathrm{E}-10$ & -22401.17 \\
\hline TOL & 1.50 & $1.00 \mathrm{E}-10$ & -23088.56 \\
\hline TON & 1.50 & $1.00 \mathrm{E}-10$ & -21378.75 \\
\hline TSC & 1.50 & $1.00 \mathrm{E}-10$ & -22177.73 \\
\hline TUN & 1.50 & $1.00 \mathrm{E}-10$ & -21975.65 \\
\hline UEI & 1.50 & $1.00 \mathrm{E}-10$ & -21926.35 \\
\hline UFI & 1.50 & $1.00 \mathrm{E}-10$ & -23338.93 \\
\hline UOS & 1.50 & $1.00 \mathrm{E}-10$ & -21762.62 \\
\hline UOZ & 1.50 & $1.00 \mathrm{E}-10$ & -22595.10 \\
\hline USI & 1.50 & $1.00 \mathrm{E}-10$ & -22055.16 \\
\hline UTL & 1.50 & $1.00 \mathrm{E}-10$ & -21437.77 \\
\hline UWY & 1.50 & $1.00 \mathrm{E}-10$ & -22153.35 \\
\hline VET & 1.50 & $1.00 \mathrm{E}-10$ & -19819.73 \\
\hline VFI & 1.50 & $1.00 \mathrm{E}-10$ & -20055.34 \\
\hline VNI & 1.50 & $1.00 \mathrm{E}-10$ & -20221.65 \\
\hline VSV & 1.50 & $1.00 \mathrm{E}-10$ & -20652.75 \\
\hline
\end{tabular}




\begin{tabular}{cccc} 
WEI & 1.50 & $1.00 \mathrm{E}-10$ & -21158.44 \\
YUG & 1.50 & $1.00 \mathrm{E}-10$ & -21359.47 \\
ZON & 1.50 & $1.00 \mathrm{E}-10$ & -22720.44 \\
\hline
\end{tabular}

Table S4: Fitted dual-site adsorption isotherm parameters for $\mathrm{N}_{2}$ adsorption on zeolites.

\section{S5.3 $\mathrm{CH}_{4}$ adsorption isotherm parameters on single-site model}

\begin{tabular}{cccc}
\hline Adsorbent & $\begin{array}{c}m_{2,1} \\
\left(\mathrm{~mol} \mathrm{~kg}^{-1}\right)\end{array}$ & $\begin{array}{c}b_{2,1} \\
(\mathrm{~Pa}-1)\end{array}$ & $\begin{array}{c}\Delta U_{2,1} \\
(\mathrm{~kJ} \mathrm{~mol}\end{array}$ \\
\hline ABW & 2.76 & $1.00 \mathrm{E}-10$ & -15183.93 \\
ACO & 5.00 & $1.00 \mathrm{E}-10$ & -19556.12 \\
AEI & 4.25 & $1.00 \mathrm{E}-10$ & -23884.70 \\
AEL & 1.66 & $1.00 \mathrm{E}-10$ & -26280.46 \\
AEN & 1.93 & $1.00 \mathrm{E}-10$ & -16629.44 \\
AET & 2.76 & $1.00 \mathrm{E}-10$ & -21102.34 \\
AFG & 2.05 & $1.00 \mathrm{E}-10$ & -26978.57 \\
AFI & 1.95 & $1.00 \mathrm{E}-10$ & -24794.43 \\
AFN & 1.95 & $1.00 \mathrm{E}-10$ & -24807.77 \\
AFO & 1.67 & $1.00 \mathrm{E}-10$ & -26239.62 \\
AFR & 5.00 & $1.00 \mathrm{E}-10$ & -22111.67 \\
AFS & 5.00 & $1.00 \mathrm{E}-10$ & -22208.46 \\
AFT & 3.88 & $1.00 \mathrm{E}-10$ & -24032.56 \\
AFX & 3.94 & $1.00 \mathrm{E}-10$ & -23930.13 \\
AFY & 5.00 & $1.00 \mathrm{E}-10$ & -22460.55 \\
AHT & 4.71 & $1.00 \mathrm{E}-10$ & -24536.77 \\
ANA & 1.00 & $1.00 \mathrm{E}-10$ & -13197.90 \\
APC & 4.48 & $1.00 \mathrm{E}-10$ & -15663.98 \\
APD & 4.94 & $1.00 \mathrm{E}-10$ & -23771.21 \\
AST & 2.47 & $1.00 \mathrm{E}-10$ & -27407.30 \\
ASV & 2.06 & $1.00 \mathrm{E}-10$ & -26349.01 \\
ATN & 2.28 & $1.00 \mathrm{E}-10$ & -26741.24 \\
ATO & 1.51 & $1.00 \mathrm{E}-10$ & -26186.31 \\
ATS & 3.31 & $1.00 \mathrm{E}-10$ & -22798.33 \\
ATT & 3.21 & $1.00 \mathrm{E}-10$ & -25422.62 \\
ATV & 3.25 & $1.00 \mathrm{E}-10$ & -23027.88 \\
AWO & 3.22 & $1.00 \mathrm{E}-10$ & -24440.56 \\
AWW & 1.84 & $1.00 \mathrm{E}-10$ & -27388.57 \\
BCT & 3.16 & $1.00 \mathrm{E}-10$ & -17470.73 \\
BEA & 4.16 & $1.00 \mathrm{E}-10$ & -23568.42 \\
BEC & 3.93 & $1.00 \mathrm{E}-10$ & -23735.77 \\
BIK & 1.74 & $1.00 \mathrm{E}-10$ & -16614.89 \\
BOF & 3.00 & $1.00 \mathrm{E}-10$ & -26217.01 \\
BOG & 4.00 & $1.00 \mathrm{E}-10$ & -23379.06 \\
BOZ & 5.00 & $1.00 \mathrm{E}-10$ & -22494.47 \\
BPH & 5.00 & $1.00 \mathrm{E}-10$ & -22301.68
\end{tabular}




$\begin{array}{clll}\text { BRE } & 2.19 & 1.00 \mathrm{E}-10 & -26869.99 \\ \text { CAN } & 2.13 & 1.00 \mathrm{E}-10 & -25139.41 \\ \text { CAS } & 3.06 & 1.00 \mathrm{E}-10 & -24686.02 \\ \text { CDO } & 1.96 & 1.00 \mathrm{E}-10 & -27038.91 \\ \text { CFI } & 2.55 & 1.00 \mathrm{E}-10 & -22396.00 \\ \text { CGF } & 1.93 & 1.00 \mathrm{E}-10 & -27729.30 \\ \text { CGS } & 2.32 & 1.00 \mathrm{E}-10 & -25847.38 \\ \text { CHA } & 3.65 & 1.00 \mathrm{E}-10 & -24537.00 \\ \text { CZP } & 2.21 & 1.00 \mathrm{E}-10 & -20278.22 \\ \text { DAC } & 3.51 & 1.00 \mathrm{E}-10 & -25340.42 \\ \text { DDR } & 2.84 & 1.00 \mathrm{E}-10 & -27384.09 \\ \text { DFO } & 4.75 & 1.00 \mathrm{E}-10 & -22925.89 \\ \text { DFT } & 4.98 & 1.00 \mathrm{E}-10 & -23781.04 \\ \text { DOH } & 3.53 & 1.00 \mathrm{E}-10 & -29354.14 \\ \text { DON } & 3.57 & 1.00 \mathrm{E}-10 & -21087.49 \\ \text { EAB } & 2.94 & 1.00 \mathrm{E}-10 & -26622.48 \\ \text { EDI } & 3.66 & 1.00 \mathrm{E}-10 & -25933.39 \\ \text { EMT } & 3.62 & 1.00 \mathrm{E}-10 & -25551.10 \\ \text { EON } & 2.31 & 1.00 \mathrm{E}-10 & -26383.71 \\ \text { EPI } & 3.27 & 1.00 \mathrm{E}-10 & -25761.97 \\ \text { ERI } & 2.77 & 1.00 \mathrm{E}-10 & -25656.49 \\ \text { ESV } & 1.73 & 1.00 \mathrm{E}-10 & -28119.42 \\ \text { ETR } & 2.83 & 1.00 \mathrm{E}-10 & -24717.41 \\ \text { EUO } & 2.30 & 1.00 \mathrm{E}-10 & -25033.40 \\ \text { EZT } & 2.82 & 1.00 \mathrm{E}-10 & -25160.45 \\ \text { FAR } & 2.30 & 1.00 \mathrm{E}-10 & -28532.78 \\ \text { FAU } & 3.39 & 1.00 \mathrm{E}-10 & -25699.22 \\ \text { FER } & 2.51 & 1.00 \mathrm{E}-10 & -27103.54 \\ \text { FRA } & 3.17 & 1.00 \mathrm{E}-10 & -27289.97 \\ \text { GIS } & 5.00 & 1.00 \mathrm{E}-10 & -22301.75 \\ \text { GIU } & 1.98 & 1.00 \mathrm{E}-10 & -28153.54 \\ \text { GME } & 4.29 & 1.00 \mathrm{E}-10 & -23306.62 \\ \text { GON } & 1.62 & 1.00 \mathrm{E}-10 & -25326.67 \\ \text { GOO } & 2.24 & 1.00 \mathrm{E}-10 & -22000.54 \\ \text { HWU } & 3.09 & 1.00 \mathrm{E}-10 & -25922.08 \\ \text { IFR } & 2.61 & 1.00 \mathrm{E}-10 & -25395.07 \\ \text { IHW } & 1.72 & 1.00 \mathrm{E}-10 & -26208.98 \\ \text { IMF } & 3.11 & 1.00 \mathrm{E}-10 & -25284.49 \\ \text { IRR } & 5.00 & 1.00 \mathrm{E}-10 & -21304.01 \\ \text { ISV } & 4.20 & 1.00 \mathrm{E}-10 & -23223.37 \\ \text { ITE } & 4.21 & 1.00 \mathrm{E}-10 & -23793.91 \\ \text { ITH } & 2.66 & 1.00 \mathrm{E}-10 & -24936.31 \\ \text { ITR } & 2.67 & 1.00 \mathrm{E}-10 & -24930.16 \\ \text { ITW } & 3.86 & 1.00 \mathrm{E}-10 & -22277.42 \\ & 3.98 & 1.00 \mathrm{E}-10 & -23777.39 \\ \text { IWS } & 5.00 & 1.00 \mathrm{E}-10 & -22441.03 \\ \text { IT-10 } & -21455.80\end{array}$




\begin{tabular}{|c|c|c|c|}
\hline IWW & 3.04 & $1.00 \mathrm{E}-10$ & -24401.52 \\
\hline JBW & 2.01 & $1.00 \mathrm{E}-10$ & -18395.74 \\
\hline JOZ & 3.84 & $1.00 \mathrm{E}-10$ & -22413.32 \\
\hline JRY & 3.13 & $1.00 \mathrm{E}-10$ & -23782.00 \\
\hline JSN & 2.73 & $1.00 \mathrm{E}-10$ & -26583.68 \\
\hline JST & 5.00 & $1.00 \mathrm{E}-10$ & -22236.71 \\
\hline JSW & 1.64 & $1.00 \mathrm{E}-10$ & -26761.60 \\
\hline KFI & 3.74 & $1.00 \mathrm{E}-10$ & -23680.63 \\
\hline LAU & 2.45 & $1.00 \mathrm{E}-10$ & -26506.59 \\
\hline LEV & 2.35 & $1.00 \mathrm{E}-10$ & -27010.32 \\
\hline LIO & 3.20 & $1.00 \mathrm{E}-10$ & -26635.53 \\
\hline LOS & 2.42 & $1.00 \mathrm{E}-10$ & -27430.83 \\
\hline LOV & 2.89 & $1.00 \mathrm{E}-10$ & -23844.77 \\
\hline LTA & 3.93 & $1.00 \mathrm{E}-10$ & -25737.45 \\
\hline LTF & 1.79 & $1.00 \mathrm{E}-10$ & -27308.89 \\
\hline LTJ & 2.31 & $1.00 \mathrm{E}-10$ & -16289.33 \\
\hline LTL & 3.41 & $1.00 \mathrm{E}-10$ & -21854.55 \\
\hline LTN & 5.00 & $1.00 \mathrm{E}-10$ & -26156.89 \\
\hline MAR & 2.16 & $1.00 \mathrm{E}-10$ & -28757.34 \\
\hline MAZ & 1.88 & $1.00 \mathrm{E}-10$ & -27259.95 \\
\hline MEI & 5.00 & $1.00 \mathrm{E}-10$ & -22687.12 \\
\hline MEL & 2.95 & $1.00 \mathrm{E}-10$ & -25310.10 \\
\hline MEP & 3.03 & $1.00 \mathrm{E}-10$ & -29696.82 \\
\hline MER & 2.74 & $1.00 \mathrm{E}-10$ & -24432.82 \\
\hline MFI & 2.83 & $1.00 \mathrm{E}-10$ & -25079.85 \\
\hline MFS & 2.35 & $1.00 \mathrm{E}-10$ & -25041.31 \\
\hline $\mathrm{MON}$ & 3.19 & $1.00 \mathrm{E}-10$ & -15855.52 \\
\hline MOR & 3.18 & $1.00 \mathrm{E}-10$ & -25137.51 \\
\hline MOZ & 2.34 & $1.00 \mathrm{E}-10$ & -25022.63 \\
\hline MRE & 1.33 & $1.00 \mathrm{E}-10$ & -27172.23 \\
\hline MSE & 3.13 & $1.00 \mathrm{E}-10$ & -24361.41 \\
\hline MSO & 2.27 & $1.00 \mathrm{E}-10$ & -25133.90 \\
\hline MTF & 1.11 & $1.00 \mathrm{E}-10$ & -27528.89 \\
\hline MTN & 3.18 & $1.00 \mathrm{E}-10$ & -30000.00 \\
\hline MTT & 1.61 & $1.00 \mathrm{E}-10$ & -25777.52 \\
\hline MTW & 1.93 & $1.00 \mathrm{E}-10$ & -24546.24 \\
\hline MVY & 2.67 & $1.00 \mathrm{E}-10$ & -24930.16 \\
\hline MWW & 3.62 & $1.00 \mathrm{E}-10$ & -24719.59 \\
\hline NAB & 2.23 & $1.00 \mathrm{E}-10$ & -14485.89 \\
\hline NAT & 5.00 & $1.00 \mathrm{E}-10$ & -21329.41 \\
\hline NES & 2.95 & $1.00 \mathrm{E}-10$ & -24126.77 \\
\hline $\mathrm{NON}$ & 1.57 & $1.00 \mathrm{E}-10$ & -26698.63 \\
\hline NPO & 2.97 & $1.00 \mathrm{E}-10$ & -10464.38 \\
\hline NPT & 4.70 & $1.00 \mathrm{E}-10$ & -23126.04 \\
\hline NSI & 1.38 & $1.00 \mathrm{E}-10$ & -14017.49 \\
\hline OBW & 5.00 & $1.00 \mathrm{E}-10$ & -22965.33 \\
\hline $\mathrm{OFF}$ & 2.57 & $1.00 \mathrm{E}-10$ & -26531.22 \\
\hline
\end{tabular}




$\begin{array}{llll}\text { OSI } & 1.68 & 1.00 \mathrm{E}-10 & -24192.26 \\ \text { OSO } & 5.00 & 1.00 \mathrm{E}-10 & -22426.12 \\ \text { OWE } & 2.53 & 1.00 \mathrm{E}-10 & -26858.71 \\ \text { PAU } & 5.00 & 1.00 \mathrm{E}-10 & -23169.59 \\ \text { PCR } & 2.18 & 1.00 \mathrm{E}-10 & -26674.24 \\ \text { PHI } & 3.15 & 1.00 \mathrm{E}-10 & -24265.82 \\ \text { PON } & 3.07 & 1.00 \mathrm{E}-10 & -24110.89 \\ \text { PUN } & 4.51 & 1.00 \mathrm{E}-10 & -23670.47 \\ \text { RHO } & 5.00 & 1.00 \mathrm{E}-10 & -23369.70 \\ \text { RRO } & 3.21 & 1.00 \mathrm{E}-10 & -18632.42 \\ \text { RSN } & 2.20 & 1.00 \mathrm{E}-10 & -23036.06 \\ \text { RTE } & 1.96 & 1.00 \mathrm{E}-10 & -27017.71 \\ \text { RTH } & 3.57 & 1.00 \mathrm{E}-10 & -24291.86 \\ \text { RUT } & 1.93 & 1.00 \mathrm{E}-10 & -28394.99 \\ \text { RWR } & 4.87 & 1.00 \mathrm{E}-10 & -18900.31 \\ \text { RWY } & 5.00 & 1.00 \mathrm{E}-10 & -21524.39 \\ \text { SAF } & 1.91 & 1.00 \mathrm{E}-10 & -23734.52 \\ \text { SAO } & 5.00 & 1.00 \mathrm{E}-10 & -22328.52 \\ \text { SAS } & 3.80 & 1.00 \mathrm{E}-10 & -23846.08 \\ \text { SAT } & 2.43 & 1.00 \mathrm{E}-10 & -25950.80 \\ \text { SAV } & 4.20 & 1.00 \mathrm{E}-10 & -23422.74 \\ \text { SBE } & 5.00 & 1.00 \mathrm{E}-10 & -22319.57 \\ \text { SBN } & 5.00 & 1.00 \mathrm{E}-10 & -23512.82 \\ \text { SBS } & 5.00 & 1.00 \mathrm{E}-10 & -21384.85 \\ \text { SBT } & 5.00 & 1.00 \mathrm{E}-10 & -21383.03 \\ \text { SFE } & 2.87 & 1.00 \mathrm{E}-10 & -22832.31 \\ \text { SFF } & 2.72 & 1.00 \mathrm{E}-10 & -24763.52 \\ \text { SFG } & 2.36 & 1.00 \mathrm{E}-10 & -24666.07 \\ \text { SFH } & 3.22 & 1.00 \mathrm{E}-10 & -21715.36 \\ \text { SFN } & 3.33 & 1.00 \mathrm{E}-10 & -21660.57 \\ \text { SFO } & 5.00 & 1.00 \mathrm{E}-10 & -22081.31 \\ \text { SFS } & 3.67 & 1.00 \mathrm{E}-10 & -23979.81 \\ \text { SFV } & 4.01 & 1.00 \mathrm{E}-10 & -23966.29 \\ \text { SGT } & 2.07 & 1.00 \mathrm{E}-10 & -27082.45 \\ \text { SIV } & 4.24 & 1.00 \mathrm{E}-10 & -23156.14 \\ \text { SOD } & 2.90 & 1.00 \mathrm{E}-10 & -29110.04 \\ \text { SOF } & 4.82 & 1.00 \mathrm{E}-10 & -23057.54 \\ \text { SOS } & 2.19 & 1.00 \mathrm{E}-10 & -21540.64 \\ \text { SSF } & 3.28 & 1.00 \mathrm{E}-10 & -22631.58 \\ \text { SSY } & 2.74 & 1.00 \mathrm{E}-10 & -22872.22 \\ \text { STF } & 3.73 & 1.00 \mathrm{E}-10 & -23955.49 \\ \text { STI } & 2.84 & 1.00 \mathrm{E}-10 & -25795.16 \\ \text { STO } & 2.00 & 1.00 \mathrm{E}-10 & -24219.04 \\ \text { STW } & 2.92 & 1.00 \mathrm{E}-10 & -25162.40 \\ & 4.71 & 1.00 \mathrm{E}-10 & -24536.77 \\ \text { STR } & 3.19 & 1.00 \mathrm{E}-10 & -24920.08 \\ \text { STE } & 1.00 \mathrm{E}-10 & -24878.72\end{array}$




\begin{tabular}{cccc} 
THO & 3.81 & $1.00 \mathrm{E}-10$ & -24603.45 \\
TOL & 2.42 & $1.00 \mathrm{E}-10$ & -27526.28 \\
TON & 2.18 & $1.00 \mathrm{E}-10$ & -25246.52 \\
TSC & 1.45 & $1.00 \mathrm{E}-10$ & -26983.93 \\
TUN & 2.99 & $1.00 \mathrm{E}-10$ & -25161.77 \\
UEI & 3.24 & $1.00 \mathrm{E}-10$ & -23938.30 \\
UFI & 3.31 & $1.00 \mathrm{E}-10$ & -26651.90 \\
UOS & 3.00 & $1.00 \mathrm{E}-10$ & -24577.96 \\
UOZ & 1.96 & $1.00 \mathrm{E}-10$ & -27847.79 \\
USI & 4.43 & $1.00 \mathrm{E}-10$ & -22980.06 \\
UTL & 4.76 & $1.00 \mathrm{E}-10$ & -22138.19 \\
UWY & 3.78 & $1.00 \mathrm{E}-10$ & -24401.87 \\
VET & 1.13 & $1.00 \mathrm{E}-10$ & -25444.77 \\
VFI & 5.00 & $1.00 \mathrm{E}-10$ & -19153.53 \\
VNI & 2.20 & $1.00 \mathrm{E}-10$ & -21657.45 \\
VSV & 3.16 & $1.00 \mathrm{E}-10$ & -17470.73 \\
WEI & 5.00 & $1.00 \mathrm{E}-10$ & -14245.90 \\
YUG & 2.39 & $1.00 \mathrm{E}-10$ & -21534.58 \\
ZON & 2.19 & $1.00 \mathrm{E}-10$ & -27842.17 \\
\hline
\end{tabular}

Table S5: Fitted dual-site adsorption isotherm parameters for $\mathrm{CH}_{4}$ adsorption on zeolites.

\section{S6 ANN Model Performance Comparison}

\begin{tabular}{ccc}
\hline Network size & $\mathrm{R}^{2}$ (Training data) & $\mathrm{R}^{2}$ (Cross-validation data) \\
\hline $5-5-5$ & 0.9479 & 0.9444 \\
$10-10-10$ & 0.9903 & 0.9875 \\
$15-15-15$ & 0.9944 & 0.9889 \\
$20-20-20$ & 0.9959 & 0.9921 \\
$25-25-25$ & 0.9977 & 0.9943 \\
$30-30-30$ & 0.9983 & 0.9954 \\
$35-35-35$ & 0.9990 & 0.9957 \\
$40-40-40$ & 0.9989 & 0.9954 \\
$45-45-45$ & 0.9987 & 0.9949 \\
$50-50-50$ & 0.9982 & 0.9929 \\
\hline
\end{tabular}

Table S6: ANN Model performance on training and cross validation datasets for $\mathrm{CO}_{2} / \mathrm{N}_{2}$ separation.

\begin{tabular}{ccc}
\hline Network size & $\mathrm{R}^{2}$ (Training data) & $\mathrm{R}^{2}$ (Cross-validation data) \\
\hline $5-5-5$ & 0.9206 & 0.9107 \\
$10-10-10$ & 0.9870 & 0.9784 \\
$15-15-15$ & 0.9936 & 0.9862 \\
$20-20-20$ & 0.9963 & 0.9871
\end{tabular}




\begin{tabular}{lll}
$25-25-25$ & 0.9975 & 0.9901 \\
$30-30-30$ & 0.9980 & 0.9909 \\
$35-35-35$ & 0.9986 & 0.9915 \\
$40-40-40$ & 0.9989 & 0.9910 \\
$45-45-45$ & 0.9987 & 0.9903 \\
$50-50-50$ & 0.9992 & 0.9910 \\
\hline
\end{tabular}

Table S7: ANN Model performance on training and cross validation datasets for $\mathrm{CO}_{2} / \mathrm{CH}_{4}$ separation.

\section{S7 Material Rankings}

\section{S7.1 Performance metrics for $\mathrm{CO}_{2} / \mathrm{N}_{2}$ separation}

\begin{tabular}{|c|c|c|c|c|}
\hline Rank & Zeolite & $\begin{array}{c}\text { Dimensionless breakthrough time } \\
-\end{array}$ & $\begin{array}{l}\text { Dynamic loading capacity } \\
\qquad\left(\mathrm{mol} \mathrm{kg}^{-1}\right)\end{array}$ & $\begin{array}{c}\text { Selectivity } \\
-\end{array}$ \\
\hline 1 & WEI & 130.37 & 1.51 & 98.92 \\
\hline 2 & JBW & 116.52 & 1.18 & 51.64 \\
\hline 3 & GIS & 114.19 & 1.33 & 43.74 \\
\hline 4 & AWW & 111.34 & 1.25 & 32.89 \\
\hline 5 & LOS & 107.92 & 1.22 & 24.16 \\
\hline 6 & $\mathrm{DFT}$ & 103.81 & 1.12 & 28.84 \\
\hline 7 & ATN & 103.47 & 1.11 & 29.09 \\
\hline 8 & MAR & 101.15 & 1.09 & 21.45 \\
\hline 9 & SIV & 100.37 & 1.17 & 40.38 \\
\hline 10 & PHI & 93.68 & 1.09 & 36.24 \\
\hline 11 & MON & 91.55 & 0.99 & 59.56 \\
\hline 12 & RWR & 90.63 & 0.90 & 41.14 \\
\hline 13 & $\mathrm{DAC}$ & 90.16 & 0.99 & 24.52 \\
\hline 14 & $\mathrm{RRO}$ & 88.03 & 0.94 & 46.63 \\
\hline 15 & $\mathrm{ABW}$ & 87.99 & 0.95 & 54.80 \\
\hline 16 & YUG & 85.13 & 0.90 & 45.85 \\
\hline 17 & MER & 85.00 & 0.99 & 32.64 \\
\hline 18 & BIK & 82.38 & 0.84 & 52.57 \\
\hline 19 & TOL & 82.00 & 0.88 & 17.20 \\
\hline 20 & EPI & 81.01 & 0.87 & 22.50 \\
\hline 21 & $\mathrm{AHT}$ & 79.96 & 0.79 & 176.17 \\
\hline 22 & $\mathrm{APC}$ & 78.58 & 0.85 & 52.73 \\
\hline 23 & HEU & 78.47 & 0.85 & 24.40 \\
\hline 24 & PAU & 76.23 & 0.86 & 30.93 \\
\hline 25 & $\mathrm{AEN}$ & 75.59 & 0.72 & 44.32 \\
\hline 26 & PUN & 73.82 & 0.94 & 30.16 \\
\hline 27 & AWO & 73.73 & 0.77 & 23.84 \\
\hline 28 & $\mathrm{ATT}$ & 73.13 & 0.82 & 26.88 \\
\hline 29 & ASV & 70.30 & 0.70 & 22.68 \\
\hline 30 & LOV & 69.85 & 0.79 & 29.09 \\
\hline
\end{tabular}




\begin{tabular}{|c|c|c|c|c|}
\hline 31 & UEI & 69.21 & 0.76 & 25.24 \\
\hline 32 & ESV & 69.00 & 0.74 & 22.14 \\
\hline 33 & ITW & 68.55 & 0.74 & 24.49 \\
\hline 34 & MOR & 68.11 & 0.77 & 18.72 \\
\hline 35 & MEP & 67.43 & 0.72 & 12.00 \\
\hline 36 & NSI & 66.85 & 0.68 & 65.77 \\
\hline 37 & $\mathrm{EON}$ & 66.61 & 0.75 & 18.58 \\
\hline 38 & UOZ & 65.87 & 0.64 & 18.45 \\
\hline 39 & AFY & 64.86 & 0.88 & 27.62 \\
\hline 40 & STW & 64.37 & 0.75 & 22.59 \\
\hline 41 & FAR & 64.36 & 0.72 & 17.53 \\
\hline 42 & SOD & 62.92 & 0.72 & 14.40 \\
\hline 43 & AFI & 62.26 & 0.68 & 29.58 \\
\hline 44 & $\mathrm{RHO}$ & 61.95 & 0.82 & 23.39 \\
\hline 45 & BRE & 61.28 & 0.64 & 18.26 \\
\hline 46 & GME & 61.13 & 0.77 & 23.17 \\
\hline 47 & $\mathrm{TSC}$ & 60.18 & 0.68 & 12.11 \\
\hline 48 & JRY & 60.12 & 0.62 & 17.23 \\
\hline 49 & APD & 59.95 & 0.64 & 18.13 \\
\hline 50 & $\mathrm{SOF}$ & 58.65 & 0.68 & 19.82 \\
\hline 51 & JST & 58.51 & 0.66 & 26.33 \\
\hline 52 & CAS & 58.28 & 0.59 & 19.54 \\
\hline 53 & $\mathrm{RSN}$ & 57.87 & 0.66 & 30.60 \\
\hline 54 & $\mathrm{AFG}$ & 57.75 & 0.65 & 25.69 \\
\hline 55 & LIO & 57.41 & 0.62 & 14.64 \\
\hline 56 & $\mathrm{RUT}$ & 57.13 & 0.60 & 15.46 \\
\hline 57 & MVY & 56.03 & 0.63 & 3166.02 \\
\hline 58 & JSW & 54.79 & 0.57 & 17.54 \\
\hline 59 & $\mathrm{AFN}$ & 53.67 & 0.59 & 29.72 \\
\hline 60 & EAB & 53.34 & 0.64 & 18.44 \\
\hline 61 & NAB & 53.28 & 0.63 & 40.75 \\
\hline 62 & UFI & 53.27 & 0.67 & 15.49 \\
\hline 63 & $\mathrm{BOZ}$ & 51.69 & 0.58 & 19.67 \\
\hline 64 & OWE & 51.36 & 0.57 & 20.80 \\
\hline 65 & $\mathrm{DOH}$ & 51.30 & 0.58 & 10.45 \\
\hline 66 & $\mathrm{ZON}$ & 51.24 & 0.54 & 12.43 \\
\hline 67 & KFI & 50.44 & 0.64 & 28.53 \\
\hline 68 & AEI & 50.12 & 0.64 & 17.94 \\
\hline 69 & GIU & 49.81 & 0.55 & 17.40 \\
\hline 70 & $\mathrm{CAN}$ & 49.13 & 0.55 & 20.62 \\
\hline 71 & AFX & 49.09 & 0.62 & 20.61 \\
\hline 72 & SFV & 48.86 & 0.55 & 12.43 \\
\hline 73 & ATS & 48.24 & 0.57 & 24.80 \\
\hline 74 & VSV & 48.20 & 0.55 & 30.24 \\
\hline 75 & GOO & 47.93 & 0.48 & 23.46 \\
\hline 76 & LTF & 47.10 & 0.53 & 17.95 \\
\hline 77 & JSN & 47.01 & 0.50 & 11.54 \\
\hline
\end{tabular}




\begin{tabular}{|c|c|c|c|c|}
\hline 78 & DDR & 46.82 & 0.50 & 11.07 \\
\hline 79 & $\mathrm{CZP}$ & 46.77 & 0.44 & 18.81 \\
\hline 80 & MTW & 46.76 & 0.49 & 23.60 \\
\hline 81 & $\mathrm{OFF}$ & 46.16 & 0.55 & 14.68 \\
\hline 82 & RTE & 46.03 & 0.51 & 17.05 \\
\hline 83 & SZR & 45.98 & 0.50 & 22.41 \\
\hline 84 & MFS & 45.45 & 0.50 & 18.58 \\
\hline 85 & TER & 45.44 & 0.51 & 14.33 \\
\hline 86 & MTF & 45.24 & 0.43 & 13.28 \\
\hline 87 & CGF & 45.06 & 0.45 & 13.07 \\
\hline 88 & LAU & 44.60 & 0.47 & 11.46 \\
\hline 89 & JOZ & 44.38 & 0.52 & 23.21 \\
\hline 90 & STI & 44.08 & 0.50 & 15.04 \\
\hline 91 & LEV & 44.04 & 0.53 & 16.59 \\
\hline 92 & $\mathrm{BPH}$ & 43.53 & 0.57 & 17.78 \\
\hline 93 & LTN & 43.40 & 0.49 & 16.03 \\
\hline 94 & SAV & 43.19 & 0.56 & 22.06 \\
\hline 95 & TON & 43.09 & 0.45 & 16.71 \\
\hline 96 & FRA & 43.04 & 0.52 & 12.43 \\
\hline 97 & STT & 42.94 & 0.48 & 15.47 \\
\hline 98 & $\mathrm{AFT}$ & 42.81 & 0.54 & 20.18 \\
\hline 99 & PCR & 42.71 & 0.48 & 11.72 \\
\hline 100 & MAZ & 42.70 & 0.49 & 18.05 \\
\hline 101 & MOZ & 41.70 & 0.47 & 17.89 \\
\hline 102 & $\mathrm{BOF}$ & 41.40 & 0.43 & 13.78 \\
\hline 103 & NPT & 41.26 & 0.58 & 23.47 \\
\hline 104 & MTT & 40.62 & 0.43 & 18.18 \\
\hline 105 & $\mathrm{ACO}$ & 39.43 & 0.46 & 19.45 \\
\hline 106 & PON & 38.95 & 0.41 & 16.27 \\
\hline 107 & $\mathrm{CDO}$ & 38.22 & 0.40 & 11.25 \\
\hline 108 & SOS & 37.62 & 0.42 & 19.42 \\
\hline 109 & MFI & 37.44 & 0.39 & 15.45 \\
\hline 110 & $\mathrm{CHA}$ & 37.36 & 0.47 & 16.21 \\
\hline 111 & EDI & 36.21 & 0.42 & 13.13 \\
\hline 112 & SFS & 35.58 & 0.41 & 13.14 \\
\hline 113 & CGS & 35.40 & 0.40 & 16.50 \\
\hline 114 & RTH & 35.36 & 0.42 & 13.05 \\
\hline 115 & SGT & 35.34 & 0.39 & 7.72 \\
\hline 116 & MTN & 34.26 & 0.39 & 8.08 \\
\hline 117 & AFR & 34.15 & 0.43 & 13.59 \\
\hline 118 & $\mathrm{SFH}$ & 33.96 & 0.39 & 17.82 \\
\hline 119 & TUN & 33.61 & 0.38 & 13.72 \\
\hline 120 & SFE & 33.16 & 0.37 & 21.13 \\
\hline 121 & $\mathrm{SBE}$ & 33.12 & 0.37 & 13.75 \\
\hline 122 & SSY & 32.99 & 0.37 & 21.21 \\
\hline 123 & IHW & 31.60 & 0.33 & 12.22 \\
\hline 124 & ITH & 31.16 & 0.34 & 12.27 \\
\hline
\end{tabular}




\begin{tabular}{|c|c|c|c|c|}
\hline 125 & SFF & 30.79 & 0.33 & 11.29 \\
\hline 126 & $\mathrm{AST}$ & 30.64 & 0.37 & 6.06 \\
\hline 127 & ERI & 30.55 & 0.36 & 13.38 \\
\hline 128 & AEL & 30.23 & 0.30 & 8.43 \\
\hline 129 & ATV & 29.95 & 0.30 & 10.37 \\
\hline 130 & USI & 29.77 & 0.36 & 12.94 \\
\hline 131 & LTA & 29.72 & 0.40 & 9.55 \\
\hline 132 & AFS & 29.39 & 0.38 & 15.56 \\
\hline 133 & OBW & 29.36 & 0.44 & 14.04 \\
\hline 134 & MWW & 29.19 & 0.35 & 11.69 \\
\hline 135 & UWY & 28.55 & 0.33 & 9.23 \\
\hline 136 & SAS & 28.48 & 0.36 & 11.07 \\
\hline 137 & FER & 28.36 & 0.31 & 11.28 \\
\hline 138 & IMF & 27.88 & 0.31 & 12.67 \\
\hline 139 & UOS & 27.58 & 0.30 & 11.24 \\
\hline 140 & MRE & 26.46 & 0.26 & 8.00 \\
\hline 141 & ITE & 26.44 & 0.32 & 12.68 \\
\hline 142 & NAT & 26.41 & 0.31 & 12.38 \\
\hline 143 & ITR & 26.31 & 0.29 & 12.72 \\
\hline 144 & THO & 25.69 & 0.31 & 11.49 \\
\hline 145 & STF & 25.32 & 0.29 & 12.24 \\
\hline 146 & MEL & 25.31 & 0.28 & 11.80 \\
\hline 147 & LTL & 25.11 & 0.29 & 17.64 \\
\hline 148 & SFN & 24.92 & 0.29 & 17.72 \\
\hline 149 & $\mathrm{EZT}$ & 24.66 & 0.27 & 8.72 \\
\hline 150 & IWR & 24.54 & 0.30 & 9.75 \\
\hline 151 & MEI & 24.23 & 0.32 & 10.32 \\
\hline 152 & SAT & 24.10 & 0.28 & 11.10 \\
\hline 153 & $\mathrm{AFO}$ & 23.90 & 0.24 & 9.59 \\
\hline 154 & DFO & 23.75 & 0.27 & 10.59 \\
\hline 155 & IFR & 23.60 & 0.26 & 8.24 \\
\hline 156 & SFO & 23.50 & 0.30 & 12.14 \\
\hline 157 & OSO & 23.11 & 0.33 & 11.83 \\
\hline 158 & MSE & 22.10 & 0.26 & 10.51 \\
\hline 159 & NES & 21.61 & 0.25 & 11.35 \\
\hline 160 & MSO & 21.59 & 0.23 & 10.00 \\
\hline 161 & $\mathrm{SBN}$ & 21.40 & 0.25 & 8.61 \\
\hline 162 & $\mathrm{BOG}$ & 21.35 & 0.25 & 9.10 \\
\hline 163 & SFG & 20.99 & 0.23 & 9.80 \\
\hline 164 & EUO & 20.90 & 0.23 & 10.29 \\
\hline 165 & ETR & 20.62 & 0.25 & 9.71 \\
\hline 166 & $\mathrm{NON}$ & 20.23 & 0.22 & 8.76 \\
\hline 167 & IWW & 19.85 & 0.23 & 9.88 \\
\hline 168 & FAU & 19.79 & 0.22 & 7.05 \\
\hline 169 & UTL & 19.46 & 0.24 & 9.25 \\
\hline 170 & EMT & 19.25 & 0.28 & 7.18 \\
\hline 171 & VNI & 18.99 & 0.21 & 12.95 \\
\hline
\end{tabular}




\begin{tabular}{llllc}
172 & ISV & 17.97 & 0.23 & 7.51 \\
173 & IWV & 17.38 & 0.20 & 8.88 \\
174 & ATO & 16.96 & 0.17 & 7.15 \\
175 & OSI & 15.84 & 0.17 & 10.46 \\
176 & BEA & 15.76 & 0.20 & 7.57 \\
177 & SAO & 15.35 & 0.21 & 6.46 \\
178 & VET & 15.31 & 0.15 & 8.34 \\
179 & BEC & 15.04 & 0.19 & 6.65 \\
180 & SSF & 14.21 & 0.16 & 6.59 \\
181 & CFI & 13.92 & 0.16 & 7.91 \\
182 & GON & 13.72 & 0.14 & 6.09 \\
183 & IWS & 13.55 & 0.15 & 6.87 \\
184 & SAF & 13.00 & 0.13 & 6.16 \\
185 & STO & 12.45 & 0.13 & 11.18 \\
186 & LTJ & 12.03 & 0.12 & 13.63 \\
187 & NPO & 11.82 & 0.12 & 5.93 \\
188 & SBS & 11.64 & 0.16 & 5.60 \\
189 & IRR & 11.00 & 0.12 & 5.61 \\
190 & AET & 10.84 & 0.11 & 5.71 \\
191 & SBT & 10.80 & 0.12 & 4.88 \\
192 & RWY & 9.94 & 0.22 & 5.73 \\
193 & VFI & 8.70 & 0.11 & 4.14 \\
194 & DON & 7.03 & 0.08 & 0.79 \\
195 & ANA & 2.63 & 0.03 & 0.16 \\
196 & BCT & 0.81 & 0.01 & \\
\hline & & & & \\
\hline
\end{tabular}

Table S8: Ranking of the 196 zeolites for $\mathrm{CO}_{2} / \mathrm{N}_{2}$ separation based on breakthrough time and dynamic loading capacity.

\section{S7.2 Performance metrics for $\mathrm{CO}_{2} / \mathrm{CH}_{4}$ separation}

\begin{tabular}{ccccc}
\hline & & Dimensionless breakthrough time & $\begin{array}{c}\text { Dynamic loading capacity } \\
\left(\mathrm{mol} \mathrm{kg}^{-1}\right)\end{array}$ & $\begin{array}{c}\text { Selectivity } \\
\text { Rank }\end{array}$ \\
\cline { 3 - 4 } & Zeolite & - & 1.77 & 12.06 \\
2 & GIS & 114.20 & 1.54 & 10.37 \\
3 & SIV & 99.22 & 1.36 & 5.91 \\
4 & WET & 94.39 & 1.43 & 323.81 \\
5 & MAR & 92.63 & 1.26 & 4.97 \\
6 & ATN & 88.28 & 1.26 & 6.89 \\
7 & PAU & 88.06 & 1.32 & 7.01 \\
8 & DAC & 87.86 & 1.25 & 5.33 \\
9 & AWW & 85.93 & 1.28 & 8.34 \\
10 & PHI & 85.25 & 1.31 & 9.46 \\
11 & JBW & 84.46 & 1.14 & 71.22 \\
12 & LOS & 84.29 & 1.24 & 5.60 \\
13 & RWR & 81.91 & 1.07 & 25.75
\end{tabular}




\begin{tabular}{|c|c|c|c|c|}
\hline 14 & $\mathrm{ATT}$ & 80.35 & 1.20 & 5.75 \\
\hline 15 & AHT & 78.45 & 1.04 & 3.53 \\
\hline 16 & STW & 78.37 & 1.22 & 4.59 \\
\hline 17 & MER & 78.27 & 1.22 & 9.48 \\
\hline 18 & EPI & 77.96 & 1.12 & 4.68 \\
\hline 19 & $\mathrm{HEU}$ & 76.84 & 1.12 & 4.84 \\
\hline 20 & YUG & 75.66 & 1.07 & 22.80 \\
\hline 21 & PUN & 74.05 & 1.26 & 7.09 \\
\hline 22 & LOV & 73.11 & 1.11 & 9.53 \\
\hline 23 & JST & 72.52 & 1.09 & 8.16 \\
\hline 24 & RHO & 71.84 & 1.26 & 5.93 \\
\hline 25 & RRO & 71.40 & 1.02 & 45.03 \\
\hline 26 & ITW & 68.81 & 0.99 & 8.34 \\
\hline 27 & $\mathrm{MON}$ & 67.62 & 0.97 & 177.69 \\
\hline 28 & SOD & 66.49 & 1.02 & 3.21 \\
\hline 29 & ESV & 66.41 & 0.95 & 6.62 \\
\hline 30 & MEP & 66.13 & 0.94 & 2.66 \\
\hline 31 & ASV & 66.07 & 0.88 & 5.12 \\
\hline 32 & BIK & 64.82 & 0.88 & 133.93 \\
\hline 33 & AWO & 64.27 & 0.90 & 4.35 \\
\hline 34 & AFI & 63.21 & 0.92 & 8.07 \\
\hline 35 & GME & 62.49 & 1.06 & 5.67 \\
\hline 36 & MOR & 62.30 & 0.93 & 3.96 \\
\hline 37 & TOL & 62.27 & 0.89 & 3.80 \\
\hline 38 & KFI & 62.24 & 1.06 & 7.19 \\
\hline 39 & $\mathrm{EON}$ & 62.23 & 0.94 & 4.29 \\
\hline 40 & $\mathrm{ABW}$ & 62.03 & 0.90 & 210.63 \\
\hline 41 & CAS & 61.81 & 0.84 & 4.22 \\
\hline 42 & AFY & 61.72 & 1.11 & 8.04 \\
\hline 43 & BRE & 60.92 & 0.85 & 4.11 \\
\hline 44 & FAR & 60.47 & 0.90 & 4.20 \\
\hline 45 & RUT & 59.78 & 0.84 & 3.97 \\
\hline 46 & LIO & 59.42 & 0.86 & 3.14 \\
\hline 47 & $\mathrm{RSN}$ & 59.32 & 0.90 & 13.16 \\
\hline 48 & MVY & 57.89 & 0.87 & 4.59 \\
\hline 49 & UEI & 57.80 & 0.84 & 5.47 \\
\hline 50 & $\mathrm{SOF}$ & 57.69 & 0.89 & 5.07 \\
\hline 51 & $\mathrm{APC}$ & 57.17 & 0.82 & 98.88 \\
\hline 52 & $\mathrm{BOZ}$ & 56.89 & 0.85 & 4.91 \\
\hline 53 & JRY & 56.01 & 0.77 & 4.82 \\
\hline 54 & UOZ & 55.53 & 0.72 & 4.37 \\
\hline 55 & AEN & 54.72 & 0.69 & 103.43 \\
\hline 56 & $\mathrm{AFN}$ & 53.98 & 0.79 & 8.08 \\
\hline 57 & UFI & 53.90 & 0.90 & 3.20 \\
\hline 58 & $\mathrm{APD}$ & 53.60 & 0.76 & 3.35 \\
\hline 59 & SAV & 53.52 & 0.93 & 5.45 \\
\hline 60 & ATS & 52.70 & 0.83 & 6.86 \\
\hline
\end{tabular}




\begin{tabular}{|c|c|c|c|c|}
\hline 61 & $\mathrm{AFT}$ & 52.55 & 0.89 & 4.73 \\
\hline 62 & $\mathrm{AFX}$ & 52.40 & 0.89 & 4.86 \\
\hline 63 & $\mathrm{NPT}$ & 52.14 & 0.99 & 5.57 \\
\hline 64 & MTW & 50.70 & 0.71 & 5.86 \\
\hline 65 & AEI & 50.33 & 0.85 & 4.15 \\
\hline 66 & $\mathrm{TSC}$ & 49.99 & 0.75 & 3.52 \\
\hline 67 & EAB & 49.63 & 0.79 & 4.13 \\
\hline 68 & $\mathrm{LTN}$ & 49.61 & 0.75 & 2.15 \\
\hline 69 & NSI & 49.37 & 0.67 & 376.69 \\
\hline 70 & PON & 48.94 & 0.68 & 3.97 \\
\hline 71 & $\mathrm{DOH}$ & 48.53 & 0.73 & 2.15 \\
\hline 72 & SZR & 47.86 & 0.69 & 5.22 \\
\hline 73 & $\mathrm{ZON}$ & 47.83 & 0.67 & 2.91 \\
\hline 74 & JOZ & 47.50 & 0.74 & 6.14 \\
\hline 75 & LTF & 46.98 & 0.71 & 4.49 \\
\hline 76 & MFI & 46.19 & 0.64 & 3.38 \\
\hline 77 & $\mathrm{CZP}$ & 45.64 & 0.58 & 12.77 \\
\hline 78 & $\mathrm{SFV}$ & 45.45 & 0.68 & 2.97 \\
\hline 79 & TER & 45.45 & 0.68 & 3.03 \\
\hline 80 & GOO & 45.19 & 0.61 & 9.11 \\
\hline 81 & $\mathrm{AFG}$ & 45.07 & 0.68 & 6.49 \\
\hline 82 & JSN & 44.98 & 0.64 & 2.48 \\
\hline 83 & FRA & 44.71 & 0.73 & 2.57 \\
\hline 84 & STI & 44.55 & 0.68 & 3.45 \\
\hline 85 & $\mathrm{STT}$ & 44.48 & 0.67 & 3.58 \\
\hline 86 & $\mathrm{CAN}$ & 44.26 & 0.67 & 6.71 \\
\hline 87 & CHA & 44.24 & 0.75 & 3.82 \\
\hline 88 & MOZ & 43.62 & 0.66 & 4.18 \\
\hline 89 & MFS & 43.60 & 0.64 & 4.29 \\
\hline 90 & $\mathrm{BPH}$ & 43.40 & 0.75 & 4.48 \\
\hline 91 & OFF & 43.39 & 0.69 & 3.33 \\
\hline 92 & DDR & 42.95 & 0.61 & 2.61 \\
\hline 93 & $\mathrm{ACO}$ & 42.32 & 0.65 & 10.21 \\
\hline 94 & NAB & 41.32 & 0.65 & 209.77 \\
\hline 95 & $\mathrm{LAU}$ & 41.15 & 0.58 & 2.49 \\
\hline 96 & MTF & 40.42 & 0.51 & 3.93 \\
\hline 97 & VSV & 40.23 & 0.61 & 43.36 \\
\hline 98 & TON & 39.82 & 0.56 & 3.77 \\
\hline 99 & SBE & 37.82 & 0.57 & 3.31 \\
\hline 100 & SFE & 37.62 & 0.57 & 5.85 \\
\hline 101 & SSY & 37.45 & 0.56 & 6.06 \\
\hline 102 & $\mathrm{CDO}$ & 36.87 & 0.52 & 2.54 \\
\hline 103 & PCR & 36.80 & 0.55 & 2.54 \\
\hline 104 & TUN & 36.12 & 0.54 & 2.96 \\
\hline 105 & OWE & 35.82 & 0.53 & 4.65 \\
\hline 106 & RTE & 35.66 & 0.53 & 4.78 \\
\hline 107 & GIU & 35.44 & 0.53 & 4.53 \\
\hline
\end{tabular}




\begin{tabular}{|c|c|c|c|c|}
\hline 108 & RTH & 35.37 & 0.56 & 3.05 \\
\hline 109 & LEV & 34.96 & 0.56 & 4.39 \\
\hline 110 & JSW & 34.60 & 0.48 & 4.69 \\
\hline 111 & SFS & 34.16 & 0.52 & 2.76 \\
\hline 112 & ITE & 33.68 & 0.54 & 2.80 \\
\hline 113 & CGS & 33.44 & 0.50 & 4.05 \\
\hline 114 & USI & 33.31 & 0.53 & 3.14 \\
\hline 115 & NAT & 33.00 & 0.52 & 4.55 \\
\hline 116 & $\mathrm{STF}$ & 32.73 & 0.49 & 2.79 \\
\hline 117 & SOS & 32.00 & 0.48 & 9.43 \\
\hline 118 & LTA & 31.92 & 0.57 & 1.91 \\
\hline 119 & AFR & 31.80 & 0.53 & 3.64 \\
\hline 120 & AFS & 31.76 & 0.55 & 4.03 \\
\hline 121 & SGT & 31.50 & 0.46 & 2.02 \\
\hline 122 & LTL & 31.47 & 0.48 & 4.89 \\
\hline 123 & ITH & 31.18 & 0.45 & 2.88 \\
\hline 124 & $\mathrm{BOF}$ & 30.37 & 0.42 & 2.69 \\
\hline 125 & ATV & 29.94 & 0.40 & 2.33 \\
\hline 126 & $\mathrm{SFF}$ & 29.91 & 0.43 & 2.72 \\
\hline 127 & THO & 29.88 & 0.48 & 2.40 \\
\hline 128 & ERI & 29.80 & 0.47 & 3.09 \\
\hline 129 & SAS & 29.23 & 0.50 & 2.62 \\
\hline 130 & UWY & 28.93 & 0.45 & 1.93 \\
\hline 131 & UOS & 28.50 & 0.41 & 2.45 \\
\hline 132 & MEL & 28.33 & 0.42 & 2.49 \\
\hline 133 & $\mathrm{DFO}$ & 28.26 & 0.42 & 2.51 \\
\hline 134 & ITR & 28.14 & 0.41 & 2.99 \\
\hline 135 & MTT & 28.02 & 0.39 & 4.73 \\
\hline 136 & $\mathrm{IMF}$ & 27.95 & 0.42 & 2.62 \\
\hline 137 & $\mathrm{SFH}$ & 27.49 & 0.42 & 5.45 \\
\hline 138 & OBW & 27.23 & 0.54 & 3.42 \\
\hline 139 & $\mathrm{AST}$ & 27.16 & 0.44 & 1.59 \\
\hline 140 & $\mathrm{SFN}$ & 26.94 & 0.41 & 5.34 \\
\hline 141 & IWR & 26.03 & 0.42 & 2.22 \\
\hline 142 & MTN & 25.04 & 0.38 & 1.87 \\
\hline 143 & AEL & 24.83 & 0.33 & 1.99 \\
\hline 144 & MRE & 24.48 & 0.32 & 1.86 \\
\hline 145 & MWW & 24.44 & 0.39 & 2.54 \\
\hline 146 & NES & 24.35 & 0.38 & 2.76 \\
\hline 147 & MEI & 24.30 & 0.42 & 2.49 \\
\hline 148 & SBN & 24.25 & 0.38 & 1.90 \\
\hline 149 & MSE & 23.97 & 0.37 & 2.40 \\
\hline 150 & IHW & 23.90 & 0.33 & 3.19 \\
\hline 151 & EDI & 23.84 & 0.37 & 2.33 \\
\hline 152 & EZT & 23.82 & 0.35 & 1.89 \\
\hline 153 & OSO & 23.38 & 0.45 & 3.25 \\
\hline 154 & $\mathrm{SFO}$ & 23.37 & 0.39 & 3.21 \\
\hline
\end{tabular}




\begin{tabular}{|c|c|c|c|c|}
\hline 155 & FER & 23.08 & 0.33 & 2.49 \\
\hline 156 & IFR & 22.62 & 0.34 & 1.96 \\
\hline 157 & VNI & 21.39 & 0.31 & 5.23 \\
\hline 158 & EUO & 20.79 & 0.31 & 2.50 \\
\hline 159 & MSO & 20.40 & 0.29 & 2.32 \\
\hline 160 & MAZ & 19.39 & 0.30 & 4.55 \\
\hline 161 & IWW & 19.27 & 0.29 & 2.26 \\
\hline 162 & UTL & 18.45 & 0.30 & 2.26 \\
\hline 163 & SAT & 18.25 & 0.28 & 2.57 \\
\hline 164 & CGF & 18.24 & 0.24 & 2.95 \\
\hline 165 & BEA & 17.58 & 0.29 & 1.70 \\
\hline 166 & FAU & 17.20 & 0.26 & 1.48 \\
\hline 167 & BOG & 16.50 & 0.26 & 2.09 \\
\hline 168 & SFG & 16.13 & 0.23 & 2.22 \\
\hline 169 & IWV & 14.96 & 0.22 & 2.50 \\
\hline 170 & OSI & 14.29 & 0.20 & 2.83 \\
\hline 171 & $\mathrm{BEC}$ & 11.77 & 0.20 & 1.51 \\
\hline 172 & ETR & 11.63 & 0.19 & 2.29 \\
\hline 173 & LTJ & 11.40 & 0.16 & 18.98 \\
\hline 174 & EMT & 11.37 & 0.22 & 1.49 \\
\hline 175 & ISV & 11.00 & 0.19 & 1.77 \\
\hline 176 & IWS & 10.97 & 0.16 & 1.73 \\
\hline 177 & $\mathrm{AFO}$ & 10.73 & 0.14 & 2.20 \\
\hline 178 & VET & 9.94 & 0.13 & 2.19 \\
\hline 179 & $\mathrm{SAO}$ & 9.57 & 0.17 & 1.67 \\
\hline 180 & SAF & 9.46 & 0.13 & 1.43 \\
\hline 181 & IRR & 9.19 & 0.14 & 1.82 \\
\hline 182 & SBT & 8.97 & 0.13 & 1.72 \\
\hline 183 & CFI & 8.80 & 0.13 & 2.28 \\
\hline 184 & NPO & 8.76 & 0.12 & 97.49 \\
\hline 185 & SBS & 8.58 & 0.16 & 1.79 \\
\hline 186 & SSF & 8.51 & 0.13 & 1.67 \\
\hline 187 & STO & 8.44 & 0.11 & 1.30 \\
\hline 188 & GON & 8.00 & 0.11 & 1.43 \\
\hline 189 & RWY & 7.99 & 0.24 & 1.89 \\
\hline 190 & AET & 6.48 & 0.09 & 1.62 \\
\hline 191 & $\mathrm{NON}$ & 5.92 & 0.09 & 2.32 \\
\hline 192 & VFI & 5.80 & 0.10 & 2.35 \\
\hline 193 & ATO & 4.37 & 0.06 & 1.59 \\
\hline 194 & $\mathrm{DON}$ & 1.95 & 0.03 & 1.14 \\
\hline 195 & ANA & 0.73 & 0.01 & 6.79 \\
\hline 196 & $\mathrm{BCT}$ & -0.17 & 0.00 & 0.24 \\
\hline
\end{tabular}

Table S9: Ranking of the 196 zeolites for $\mathrm{CO}_{2} / \mathrm{CH}_{4}$ separation. 


\section{References}

[1] Lu Wang, Zhen Liu, Ping Li, Jianguo Yu, and Alirio E Rodrigues. Experimental and modeling investigation on post-combustion carbon dioxide capture using zeolite 13x-apg by hybrid vtsa process. Chemical Engineering Journal, 197:151-161, 2012.

[2] Wangyun Won, Seunghun Lee, and Kwang-Soon Lee. Modeling and parameter estimation for a fixed-bed adsorption process for $\mathrm{CO}_{2}$ capture using zeolite 13X. Separation and purification technology, 85:120-129, 2012. 